\title{
Structure and water attachment rates of ice in the atmosphere: role of nitrogen
}

\author{
Pablo Llombart ${ }^{\dagger \ddagger}$, Ramon M. Bergua ${ }^{\ddagger}$, Eva G. Noya ${ }^{\dagger}$, and Luis G. MacDowell ${ }^{\ddagger}, *$ \\ † Instituto de Química Física Rocasolano , CSIC, \\ Calle Serrano 119, 28006 Madrid, Spain and \\ $\ddagger$ Departamento de Química-Física (Unidad de I+D+i Asociada al CSIC), \\ Facultad de Ciencias Químicas, Universidad Complutense de Madrid, 28040 Madrid, Spain*
}

\begin{abstract}
In this work we perform computer simulations of the ice surface in order to elucidate the role of nitrogen in the crystal growth rates and crystal habits of snow in the atmosphere. In pure water vapor at temperatures typical of ice crystal formation in cirrus clouds, we find that basal and primary prismatic facets exhibit a layer of premelted ice, with thickness in the subnanometer range. For partial pressures of 1 bar, well above the expected values in the troposphere, we find that only small amounts of nitrogen are adsorbed. The adsorption takes place onto the premelted surface, and hardly any nitrogen dissolves within the premelting film. The premelting film thickness does not change either. We quantify the resulting change of the ice/vapor surface tension to be in the hundredth of $\mathrm{mN} / \mathrm{m}$ and find that the structure of the pristine ice surface is not changed in a significant manner. We perform a trajectory analysis of colliding water molecules, and find that the attachment rates from direct ballistic collision are very close to unity irrespective of the nitrogen pressure. Nitrogen is however at sufficient density to deflect a fraction of trajectories with smaller distance than the mean free path. Our results show explicitly that the reported differences in growth rates measured in pure water vapor and a controlled nitrogen atmosphere are not related to a significant disruption of the ice surface due to nitrogen adsorption. On the contrary, we show clearly from our trajectory analysis that nitrogen slows down the crystal growth rates due to collisions between water molecules with bulk nitrogen gas. This clarifies the long standing controversy of the role of inert gases on crystal growth rates and demonstrates their influence is solely related to the diffusion limited flow of water vapor across the gas phase.
\end{abstract}

*Igmac@quim.ucm.es 


\section{INTRODUCTION}

Modeling of radiation processes in clouds is an essential requisite for the prediction of climate change.[1, 2] In the troposphere, ice is a widespread component, as cirrus clouds cover about $30 \%$ of the mid latitudes at any given time.[3] The ice grains that make these cirrus clouds, whether in mono-crystalline form or as crystal aggregates play a crucial role on the earth's climate.[4-8] They account for a significant amount of the radiation budget,[4-7] and concentrate airborne chemicals with an important contribution to the atmosphere's chemistry.[9] However, the underlying microphysics of ice crystallites is a major source of uncertainty for climate change models, while the mechanism for ice growth and surface activity remains poorly understood.[1]

In view of this situation, a great number of recent computer simulation studies seek to characterize the ice surface and shed light onto growth mechanisms.[10-18] In most such studies, a bulk ice sample is placed either in vacuum or within a saturated water vapor atmosphere, and only rarely is the interaction of atmospheric gases considered explicitly.[19, 20] Of course, acidic gases and polar organic molecules adsorbed on the ice surface are expected to play a crucial impact on atmospheric chemistry.[9, 20-22] However, they are usually only found in trace amounts. As an example, formic acid, which is one of the most abundant strong short chain acid in the atmosphere is found in a mixing ratio of 2 parts per billion in the boundary layer air.[22] Therefore, the study of pristine ice surfaces as an initial starting point is warranted.[21]

On the contrary, the two major components of the atmosphere, nitrogen and oxygen, have far less affinity for ice, but are found at concentrations two orders of magnitude larger than saturated water vapor. Although laboratory studies are often performed on a controlled atmosphere of nitrogen gas to avoid possible bias,[23-27] the fact is that their role on the structure and growth rates remains largely unknown.[20]

A chemical physicist's first guess clearly indicates that an inert gas such as nitrogen should have a very small impact on the properties of the ice surface. Indeed, reported thermodynamic data on surface tension and phase coexistence data for water essentially ignore the role of the surrounding atmosphere. From Henry's constant data,[28] it is expected that the concentration of nitrogen in water at $0 \mathrm{C}$ and one bar is not more than $10^{-3} \mathrm{~mol} / \mathrm{L}$, and likely far smaller in ice. Furthermore, BET adsorption studies of nitrogen on ice consistently yield small enthalpies of adsorption of about $\Delta H_{a d s}=8.1 \mathrm{~kJ} / \mathrm{mol}$ at $77 \mathrm{~K}$.[29-31] Measurements using other apolar gases such as methane yield similar results.[32, 33] Furthermore, Temperature Programmed Desorption experiments show that monolayers of nitrogen on ice have fully desorbed at temperatures below $50 \mathrm{~K}$, corresponding to estimated adsorption energies of $9.6 \mathrm{~kJ} / \mathrm{mol}$ at most.[34-36]

Intriguingly, laboratory studies of surface structure and crystal growth rates have recurrently reported significant differences between experiments performed on either a controlled nitrogen or pure water vapor atmosphere.[37-42] 
In experiments of ice crystals grown from the vapor, it is well known that the presence of significant amounts of nitrogen gas largely slows down the crystal growth rates.[43, 44] However, it has been usually interpreted that this is not the effect of a significant perturbation of the ice surface, but rather, the effect of diffusion limited growth.[45, 46] This occurs when the slow diffusion rate of water vapor in air is unable to reestablish the depleted ambient vapor density close to the growing ice surface. As a result, the interpretation of growth measurements requires to distinguish the local surface saturation from the asymptotic bulk vapor saturation. In view of the difficulty to accurately estimate the full density field at the crystal, standard experiments aimed at unraveling the correct surface attachment kinetics have rather been performed in low pressure chambers with as small nitrogen as possible, under the assumption that nitrogen plays no other role than decreasing the diffusion coefficient of water vapor.[46-50]

However, some studies on crystal growth, with explicit account of diffusion resistance of air reported that the surface saturation corrected growth rates were strongly influenced by nitrogen,[37-39] decreasing the surface kinetics selectively on the prismatic facet by factors as large as $1 / 100$ at $\mathrm{T}=-11 \mathrm{C}$, and $1 / 20$ at $\mathrm{T}=-1 / 20$ at $15 \mathrm{C}$ compared to experiments in pure water vapor.[37, 39] In fact, it was claimed that adsorption of nitrogen was essential to explain the anisotropic growth of ice crystals as described in the Nakaya diagram. Particularly, Beckmann and collaborators postulated a nitrogen poisoning mechanism that is well known in catalysis.[38] Unfortunately, this explanation assumes enthalpies of adsorption ranging from 25 to $40 \mathrm{~kJ} / \mathrm{mol}$, which are very much at odds with BET isotherm experiments, recent Temperature Programmed Desorption experiments,[3436] and ab-initio calculations.[20, 29-31] On theoretical grounds, another possible role of adsorbed gases is to proliferate in between crystal steps and kinks. This can potentially change the step free energy, and as a result, change significantly the surface roughness and crystal growth rates.[51] However, none of these hypothesis seem to be consistent with later studies of crystal growth habits at reduced pressure, which seem to agree well with results for the standard Nakaya diagram,[46, 49, 52] and exhibit a distinct growth anisotropy,[46, 48-50, 53] similar to that observed in the presence of air.[54, 55] Furthermore, similar slowing down of growth rates has been confirmed with other inert gases, in support of merely a diffusion limited role on ice growth.[45, 52]

Atmospheric gases have also been postulated to play a significant role in determining the thickness of premelting films of water adsorbed onto the ice surface.[27, 40, 41, 56-59] Indeed, conflicting results for the equilibrium thickness of premelting films have regularly been found, with thicknesses differing by several orders of magnitude.[60-62] These discrepancies can be partly reconciled by taking into account that contamination can increase the thickness of the premelting film considerably.[27, 40, 41, 63] An uncontrolled exposure to the atmosphere can easily promote such contamination, and this explains why often crystal samples in air exhibit much larger film thicknesses.[41] However, claims that the thickness of premelting films diverges in the presence of nitrogen,[40] do not seem warranted in view of more recent experiments indicating a finite equilibrium thickness 
in a controlled nitrogen atmosphere.[23-26]

In view of this account, it seems clear that the role of adsorbed nitrogen on such significant atmospheric properties as the equilibrium surface structure and the crystal growth rate of ice remains largely unknown and controversial. Certainly, whatever conclusion is made from the current evidence is a result of indirect and hypothetical arguments from macroscopic samples. Computer simulations could be an invaluable tool to shed light into this complex problem, as they can elucidate the microscopic features of the crystal growth mechanism. Indeed, Libbrecht has urged for such a study, in order to clarify some conflicting results on the role of nitrogen in recent crystal growth experiments.[42, 50]

In this work we perform a computer simulation study of the ice surface under a controlled nitrogen atmosphere. In our study we first perform computer simulations of ice in presence of pure water vapor and provide a detailed structural characterization of the ice/vapor interface (section III A). We then perform additional simulations of ice under nitrogen gas and study the adsorbed layer of nitrogen (section III B), and its influence on the structural and thermodynamic properties of the ice interface (section III C). Finally, we present results on the influence of nitrogen gas in water attachment kinetics. (section IV). In section V we summarize and discuss the significance of our results. Overall we find that nitrogen gas does not significantly change the properties of the ice surface, and conclude that crystal growth rate and crystal habit differences observed for ice in nitrogen are related only to the complex diffusion limited flow of water towards the ice surface.

\section{METHODS}

\begin{tabular}{lcr}
\hline \hline Altitude $/ m$ & $T / K p \cdot 10^{-5} / \mathrm{Pa}$ \\
\hline 2750 & 270 & 0.72 \\
4250 & 260 & 0.60 \\
5800 & 250 & 0.48 \\
7310 & 240 & 0.40 \\
8840 & 230 & 0.32 \\
\hline \hline
\end{tabular}

TABLE I: Average atmospheric temperature and pressure as a function of altitude according to the International Standard Atmosphere (ISO 2533:1975) 


\section{A. Simulations}

All simulations were performed with the simulation package GROMACS[64, 65]. Simulations were carried out mostly in the NVT ensemble. Temperature was controlled using the Bussi-Donadio-Parrinello velocityrescale thermostat with a relaxation time of 1 ps[66]. In those simulations carried out in NpT ensemble, the Berendsen barostat was used to achieve the desired pressure. Periodic boundary conditions were applied on the three directions of space. Lennard-Jones interactions were truncated at a distance of $9 \AA$ and homogeneous long range corrections applied.[67, 68] Coulomb interactions were evaluated using Particle Mesh Ewald, with the real space contribution truncated also at $9 \AA$. The reciprocal space term is evaluated over a total of $80 \times 64 \times 160$ vectors in the $x, y, z$ reciprocal directions, respectively. The charge structure factors were evaluated with a grid spacing of $0.1 \mathrm{~nm}$ and a fourth order interpolation scheme. The Gaussian charge distribution width was set to $0.288146 \mathrm{~nm}$. Equations of movement were solved using constraint molecular dynamics, with the GROMACS default LINCS algorithm, the velocity-Verlet integrator and a timestep of $3 \mathrm{fs}$.

\section{B. Force Field}

Water and nitrogen were described using rigid, non-polarizable point charge models, TIP4P/Ice [69] for water and TraPPE [70] for nitrogen. TIP4P/Ice was fitted to reproduce the melting temperature of ice Ih, the densities of liquid water and ices II and V, and the stability region of ice III[69], whereas TraPPE was fitted to reproduce the vapor-liquid equilibrium of nitrogen and nitrogen mixtures [70]. It reproduces the full coexistence curve up to the critical point as well as the vapor pressures. Crossed Lennard-Jones interactions between nitrogen and water are modeled using Lorentz-Berthelot rules, with a crossed $\mathrm{LJ} \sigma$ parameter determined as the arithmetic average and the crossed $\mathrm{LJ} \epsilon$ parameter determined as the geometric mean.

Of course, the use of two widely recognized force fields for the pure components does not guarantee an accurate description of the crossed interactions, which is here a crucial issue. For this reason we have performed a number of calculations to check the validity of the model as regards $\mathrm{H}_{2} \mathrm{O}-\mathrm{N}_{2}$ interactions.

\section{1. $\mathrm{H}_{2} \mathrm{O}-\mathrm{N}_{2}$ dimer interactions}

The binding energy of $\mathrm{H}_{2} \mathrm{O}-\mathrm{N}_{2}$ dimers has been estimated by a number of authors using ab-initio, and semiempirical methods.[20, 71, 72] Using a semi-empirical method, Girardet and Toubin advocate $E_{b}=4.8 \mathrm{~kJ} / \mathrm{mol}$, for the optimized binding energy, in agreement with ab-initio MP2 results by Sadlej et al,[71] and close to a more recent MP2 calculation which provide $E_{b}=5.2 \mathrm{~kJ} / \mathrm{mol} .[72]$

We have performed an energy minimization for the gas phase $\mathrm{H}_{2} \mathrm{O}-\mathrm{N}_{2}$ dimer interactions, using GROMACS 
energy optimization module. This applies a a steepest descent algorithm to find the global energy minimum of the system. The lowest energy configuration predicted by our model corresponds to the linear nitrogen molecule aligned along an $\mathrm{OH}$ bond in water, precisely as found in electronic calculations.[72] The optimized energy yields $E_{b}=4.97 \mathrm{~kJ} / \mathrm{mol}$, which lies in between the MP2 results of Sadlej et al.[71] and Tulegenov et al.[72], and is less than $5 \%$ away from either calculation.

\section{Heat of adsorption of nitrogen on ice}

Adsorption energies of nitrogen on ice at temperatures in the range studied in this work do not seem to be available. The most significant sources of information come from the astrophysics [34-36] and geophysics community for temperatures below 100 K.[29-31] In astrophysics, temperature programed desorption experiments (TDP) in the range between 20 to about $50 \mathrm{~K}$ have been performed on both polycrystalline ice samples (PCI) and amorphous solid water (ASW).[34-36] Results show little differences between one or the other solid phase,[36] and provide average adsorption energies ranging between 9.6 [35] and $9.5 \mathrm{~kJ} / \mathrm{mols}$.[34] However, it is well known that at the low temperature of these experiments, nitrogen molecules are sufficiently mobile to surface hop to the most favorable adsorption sites before desorption. At higher temperatures, where desorption proceeds much faster, molecules do not typically have time to reach the most favorable adsorption sites and the average adsorption energy can be significantly smaller.[34-36] This can be already seen from BET experiments of $\mathrm{N}_{2}$ on snow, that are performed in the geoscience community as a probe of snow porosity at a temperature of 77 K.[29-31] Results show a very broad range of porosity depending on sample location and history, but yield fairly consistent average adsorption energies of ca. $8.1 \mathrm{~kJ} / \mathrm{mol},[29,30]$ already significantly lower than results observed for TPD in the range between 20 and $50 \mathrm{~K}$, and somewhat lower than estimates obtained from the frequency shift of the $\mathrm{OH}$ dangling bond stretch upon adsorption, which provide ca. $8.7 \mathrm{~kJ} / \mathrm{mol}$.[73] These results all fall between the $\mathrm{H}_{2} \mathrm{O}-\mathrm{N}_{2}$ dimer energy and the hydration energy of $\mathrm{N}_{2}$ in water at ambient temperature, which, from the NIST webbook is $10.8 \mathrm{~kJ} / \mathrm{mol}$. In view of this discussion, the ab-initio calculations of Manca and Allouche,[74] which suggest an energy of adsorption of barely $3.1 \mathrm{~kJ} / \mathrm{mol}$ seem to be somewhat too low.

In section III C we calculate the adsorption energies of nitrogen gas on the ice surface. Our results provide $\Delta H_{a d s}=7.4 \mathrm{~kJ} / \mathrm{mol}$ for the basal face and $\Delta H_{a d s}=6.5 \mathrm{~kJ} / \mathrm{mol}$ for the prismatic face. Bearing in mind that these results are measured from adsorption data in the range between 210 and $270 \mathrm{~K}$, i.e., $150 \mathrm{~K}$ above the BET experiments, and enthalpies of adsorption are expected to decrease with temperature, $[34,36]$ our results seem very reasonable.

Table II summarizes available experimental results for $\mathrm{H}_{2} \mathrm{O}-\mathrm{N}_{2}$ interactions relevant to our study. The agreement with results from our work is overall rather satisfactory, in view of the lack of experimental data available 
Technique Sample Temperature range / $\mathrm{K} \Delta H_{a d s} / \mathrm{kJ} \cdot \mathrm{mol}^{-1}$ Reference

\begin{tabular}{|c|c|c|c|c|}
\hline Semi-empirical & $\mathrm{N}_{2}-\mathrm{H}_{2} \mathrm{O}$ & 0 & 4.8 & [20] \\
\hline ab-initio MP2 & $\mathrm{N}_{2}-\mathrm{H}_{2} \mathrm{O}$ & 0 & 5.2 & {$[72]$} \\
\hline ab-initio MP2 & $\mathrm{N}_{2}-\mathrm{H}_{2} \mathrm{O}$ & 0 & 4.8 & {$[71]$} \\
\hline MD & $\mathrm{N}_{2}-\mathrm{H}_{2} \mathrm{O}$ & 0 & 5.0 & This work \\
\hline TPD & ASW & $20-50$ & 9.5 & [34] \\
\hline TDP & ASW & $20-50$ & 9.6 & {$[35]$} \\
\hline BET & PCI & 77 & 8.1 & [29] \\
\hline BET & PCI & 77 & 8.3 & [30] \\
\hline IRS & Ic & 96 & 8.7 & {$[73]$} \\
\hline $\mathrm{HF}$ & Ih & 0 & 3.3 & {$[74]$} \\
\hline $\mathrm{MD}$ & Ih-basal & $230-270$ & 7.4 & This work \\
\hline MD & Ih-pI & $230-270$ & 6.5 & This work \\
\hline
\end{tabular}

TABLE II: Adsorption enthalpies of $\mathrm{N}_{2}$ for solid water phases as estimated with different techniques. TDP: Temperature Programmed Desorption; BET: Brunauer-Emeret-Teller isotherms; IRS: Infrared spectroscopy; HF-MP2: Hartree Fock plust Möller Plesset perturbation theory. Samples are: ASW, Amorphous solid water; PCI: polycrystalline ice; Ic: Cubic ice. Ih: hexagonal ice.

in the range of temperatures considered here.

\section{Determination of the equation of state of nitrogen gas}

Prior to the simulations of the ice-vapor interface, we carried out a series of simulations aimed at determining the equation of state for nitrogen. This equation is later used to relate the number of adsorbed nitrogen molecules with the corresponding bulk pressure in the ice-vapor system. The simulations covered densities within $\rho=0.5-2$ $\mathrm{kg} \cdot \mathrm{m}^{-3}$ and temperatures within $T=230-270 \mathrm{~K}$ (at $5 \mathrm{~K}$ intervals), corresponding to pressures between about 0.30 to 1.1 bars that bracket the expected pressure of the International Standard Atmosphere (c.f. Tab.I) . All simulations were carried out in a cubic box of length $21.5 \mathrm{~nm}$, containing between 120 and 420 nitrogen molecules depending on density. Each of these thermodynamic states was simulated during $3 \mathrm{~ns}$ averaging every 75 ps for the calculation of thermal averages. Pressure was estimated as an ensemble average using the virial route[67]. For each isotherm, the pressure as a function of density was fitted to:

$$
P(T)=\rho k_{B} T\left(1+B_{2}(T) \rho\right)
$$


where $k_{B}$ is the Boltzmann constant and $B_{2}(T)$ is the second virial coefficient. The values of $B_{2}(T)$ obtained at each temperature were then fitted to the function $B_{2}(T)=A_{0}-A_{1} T^{-1}$. Eq. 1 together with this fit can be used to predict the pressure of nitrogen for any particular values of density and pressure within the range of simulated densities and pressures.

The equation of state data and the corresponding virial coefficients are collected in detailed form in the supplementary material. The pressure isotherms and fits to the virial coefficients are displayed in Figure 1. Whereas the empirical model employed for nitrogen was fitted at far lower temperatures to match coexistence liquid densities, we find a reasonable agreement for the Boyle temperature, which, by extrapolation from our results is $T_{B}=296 \mathrm{~K}$, compared to the reported experimental value of $T_{B}=326 \mathrm{~K}$.[75]
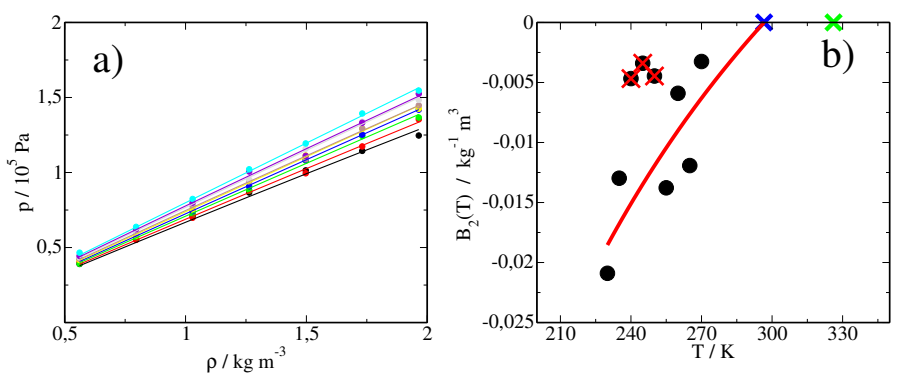

FIG. 1: (a) Pressure as a function of nitrogen density, with simulation results shown as circles. In order of decreasing slope, studied temperatures are 230 (black), 235 (red), 240 (green), 245 (blue), 250 (yellow), 255 (gray), 260 (brown), 265 (violet) and 270 (cyan) respectively. Fits to Eq. 1 for each isotherm are shown as full lines with the same color code. (b) Second Virial Coefficient, $B_{2}(T)$, as a function of temperature. Black circles correspond to the slopes obtained from the fit to the isotherms, while the red line represents the fit to the function $B 2(T)=0.06402\left(\mathrm{~kg}^{-1} \mathrm{~m}^{3}\right)-18.990\left(\mathrm{~kg}^{-1} \mathrm{~m}^{3} \mathrm{~K}\right) \mathrm{T}^{-1}\left(\mathrm{~K}^{-1}\right)=$ $A_{0}-A_{1} T^{-1}$. Symbols with red crosses were not taken into account for the fit. The blue cross is the extrapolated Boyle temperature obtained from the simulated results. The green cross corresponds to the experimental Boyle temperature.[75]

\section{Simulation of the ice-vapor interface in the presence of nitrogen}

The ice-vapor interface was built using the following procedure. We start by generating a slab of ice containing $8 \times 8 \times 5$ pseudo-orthorhombic unit cells with 16 molecules each, whence, a total of 5120 water molecules. At each temperature, the bulk solid is simulated during $6 \mathrm{~ns}$ in the NpT ensemble and $p=1$ bar. The simulation cell is then rescaled to its equilibrium value, and placed inside a larger elongated cell along the direction $z$, perpendicular to the interface. A number of nitrogen molecules are then randomly inserted in the vacuum region of the box. Simulation boxes typically had a lateral size $L_{x}=7 \mathrm{~nm}$ and $L_{y}=6 \mathrm{~nm}$. The perpendicular size was set to $L_{z}=15 \mathrm{~nm}$ for pure ice under water vapor and $L_{z}=39 \mathrm{~nm}$ for ice in a nitrogen atmosphere. For the latter simulations, the gas phase was formed with 15 to 50 nitrogen molecules. Detailed information about system size 
and dimensions are provided in the supplemental material.

The system is evolved in the NVT ensemble during $15 \mathrm{~ns}$ to allow the premelting layer to equilibrate. Averages are taken over the next $35 \mathrm{~ns}$, saving configurations every 75 ps. The density profile of the vapor phase established mainly by nitrogen molecules is measured, and these data are used to infer the ambient pressure from Eq. (1). Amounts of nitrogen are selected such that, for each temperature, the pressure ranges from the expected value for the International Standard Atmosphere (c.f. Tab.I) to a pressure somewhat above 1 bar.

The amount of nitrogen adsorbed on the ice surface at each thermodynamic state was quantified by measuring the surface excess or Gibbs adsorption:

$$
\Gamma_{\mathrm{N}_{2}}=\left(n_{\mathrm{N}_{2}}-\rho_{\mathrm{N}_{2}} V_{\mathrm{N}_{2}}\right) / A
$$

where $n_{\mathrm{N}_{2}}$ is the total number of nitrogen molecules within the simulation box, $\rho_{N 2}$ is the bulk density of nitrogen, $V_{\mathrm{N}_{2}}$ the volume of the vapor phase, and $A$ the area of the system in a plane parallel to the interface. Relative to the ice/vapor interface, the volume of the vapor phase is determined for an equimolar dividing surface as $V_{\mathrm{N}_{2}}=A\left(L_{z}-H\right)$, where $\mathrm{H}$ is the thickness of the ice slab, $H=N_{w} /\left(A \rho_{\text {ice }}\right)$ and $N_{w}$ is the total number of water molecules in the condensed phase. The change of the surface tension, $\gamma$, as a consequence of a change of the pressure induced by nitrogen is accounted for by the Gibbs equation:[76]

$$
d \gamma=-\Gamma_{\mathrm{N}_{2}} R T \frac{d p}{p}
$$

where $R$ is the gas constant, $T$ is temperature and $p$ is the partial pressure of nitrogen. At the low pressures considered in this study, the nitrogen surface excess increases linearly with pressure $\Gamma_{\mathrm{N}_{2}}=a p$, and the slope $a$ can be adjusted to the simulation data. Substitution of this expression into Eq. 4, yields:

$$
\Delta \gamma=-\Gamma_{\mathrm{N}_{2}} R T=-a R T p
$$

\section{E. Intrinsic surfaces and order parameter}

In the simulations, the ice slabs in vacuum develop a thin layer of disordered quasi-liquid like molecules. The properties of this layer are best characterized by defining ice-liquid and liquid-vapor surfaces that sandwich the premelting layer.[77, 78] The thickness of the liquid layer formed on the surface of ice can be determined from the average distance between the surfaces.

\section{Order parameter}

In order to locate the surfaces, we need first of all to establish a criterion to distinguish between solid and liquid water molecules. 
For that purpose we used the local averaged order parameter, $\bar{q}_{6}$, proposed by Lechner and Dellago [79], which averages the Steinhardt's local parameter, $q_{6}$,[80] over first neighbor shells. This order parameter adopts different values depending on the local environment of the water molecule. Solid molecules adopt large values of $\bar{q}_{6}$, whereas molecules in liquid environments exhibit low values of $\bar{q}_{6}$. Figure 2 shows distributions of the probability $P\left(\bar{q}_{6}\right)$ to observe a given value of $\bar{q}_{6}$ in either the solid or liquid phase for the temperatures studied in this work. As found in our previous work, $[81,82]$ the distribution is close to Gaussian for both the solid and liquid phases, with peaks that are well separated and show only a small region of overlap. A threshold value of $\bar{q}_{6}$ used to discriminate between solid and liquid particles is obtained by plotting the distribution of $\bar{q}_{6}$ for molecules in bulk solid and bulk liquid environments, and finding the limiting value of $\bar{q}_{6}$ that leads to minimum mislabeling, as described in Ref. 81-83. The threshold values obtained at each temperature are provided in Fig. 2. A sample configuration in which $\bar{q}_{6}$ was used to label the particles as solid or liquid is also shown in Fig. 2.

Notice that the identification of individual molecules as 'solid' or 'liquid' like is not without a degree of arbitrariness, since bulk phases can only be properly defined from ensemble averages of appropriate order parameters over long times. This difficulty is particularly significant for water, which can exhibit a large number of allotropes, and could thus have a number of different order parameters consistent with the solid phase. Particularly at the solid/liquid interface, a number of different local environments that resemble bulk ice phases have been identified based on the number of staggered and eclipsed bonds, such as hexagonal ice, cubic ice, or ice clathrate forms, as well as purely interfacial varieties such as interfacial hexagonal ice.[84] The total number of liquid-like molecules will therefore depend on the decision as to how to attribute allotropic forms different from ice Ih into 'solid-like' or 'liquid-like' environments. By using the $\bar{q}_{6}$ parameter, we are able to identify local environments consistent with hexagonal ice, but we cannot distinguish disordered liquid-like environments from other more ordered environments such as cubic ice, ice clathrates or interfacial hexagonal ice. It must therefore be understood that our choice of ice-liquid surface separates essentially bulk ice Ih from a loosely defined premelting layer which includes liquid-like environments as well as relatively ordered allotropic forms. Alternatively, using the CHILL+ algorithm, one can distinguish all such forms.[84] In the conventional application of the CHILL+ algorithm, ice clathrate and ice Ic, as well as interfacial ice Ih environments, are attributed to the solid phase, and the quasi-liquid layer is made only of the disordered molecules. Therefore, the premelting layer thickness somewhat differs, in a similar way as it does by using different experimental probes.[27, 40, 41, 58-62] Coincidentally, we have checked that the number of molecules in ice clathrate, ice Ic and interfacial ice Ih environments remains almost constant within the temperature interval studied in our work, so that the premelting layer thickness defined in either way only differs by an almost constant offset (supplemental material). For consistency with our previous work, $[77,78,81,82]$ we will therefore use the $\bar{q}_{6}$ parameter as a criterion for discriminating liquid-like from solid-like environments. 


\section{Intrinsic surface}

Once that water molecules have been identified as solid or liquid, the largest cluster is searched using a cluster analysis algorithm, in which two water molecules are considered to be nearest neighbors if the distance between their oxygen atoms is smaller than $3.5 \AA$.

The outermost layer of oxygen atoms on this cluster corresponds to water-like molecules. To locate the liquidvapor surface at a point $(x, y)$ on the plane of the interface, $h_{w v}(x, y)$ we search for all liquid like atoms within a rectangular area of $3 \sigma \times 3 \sigma$, and define the surface height as the average value of the four outermost atoms. Having found the liquid-vapor surface at that point, we then locate the solid-like atoms within a rectangular unitcell plane about $(x, y)$. The four outermost solid-like atoms within that area determine the solid-liquid surface location, $h_{i w}(x, y)$.

A local liquid-layer thickness at point $(x, y)$ can be calculated as

$$
\delta h(x, y)=\left|h_{i w}(x, y)-h_{w v}(x, y)\right|
$$

The average thickness is determined by averaging the local heights over a mesh with twice as many points as unit cells on the surface.
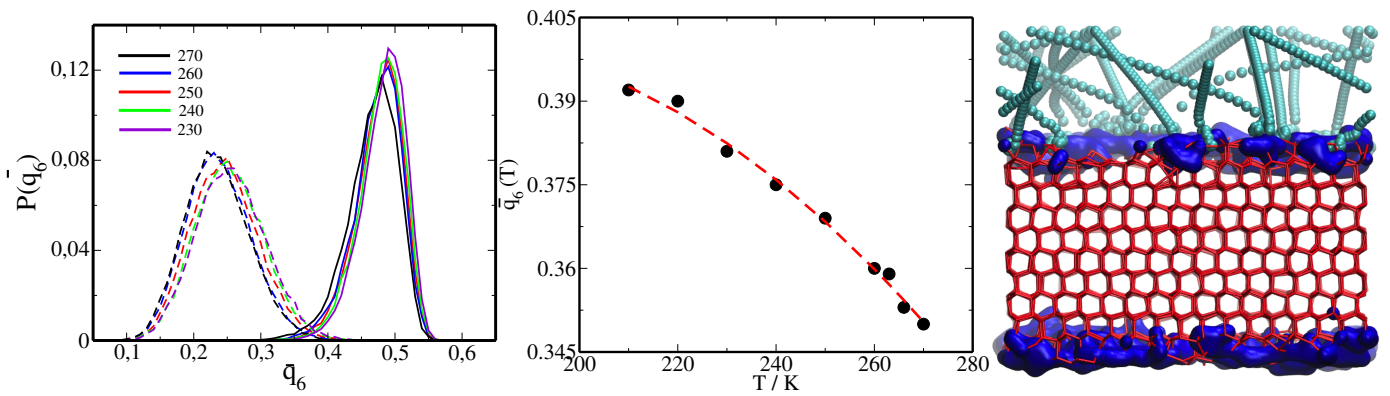

FIG. 2: Left: Probability distribution of the $\bar{q}_{6}$ on the solid (full lines) and liquid (dashed lines) phases for temperatures between $230 \mathrm{~K}$ and $270 \mathrm{~K}$ as indicated in the color code. Middle: Limiting values of $\bar{q}_{6}$ used to discriminate liquid from solid particles as a function of temperature. These values were obtained using the mislabeling criterion described by Espinosa et al. [83]. These limit values can be accurately fitted to a quadratic polynomial function: $\bar{q}_{6}(T)=0.26266+0.0016474 T-$ $4.8982 \cdot 10^{-6} T^{2}$. Right: Atomistic view of the ice-vapor interface at $\mathrm{T}=230 \mathrm{~K}$ and of the trajectory of 20 water molecules that are shot to the ice surface. Water molecules identified with $\bar{q}_{6}$ as solid are shown as red sticks, and liquid molecules as dark blue spheres. The trajectories followed by the molecules are depicted as cyan spheres.

Further information about the structure of the ice-liquid and liquid-vapor interfaces can be obtained from the density profiles measured along the direction perpendicular to the interface (i.e. along $z$ ):

$$
\rho_{\alpha}(z)=\frac{N_{\alpha}(z)}{L_{x} L_{y} \Delta z}
$$


where $\alpha$ denotes either solid-like or liquid-like molecules, and $N_{\alpha}(z)$ is the number of molecules of that type in a slice of simulation box centered at $z$ and of width $\Delta z$.

Besides analyzing the density profile as a function of the absolute $z$-coordinate measured with respect to a fixed reference system, we also evaluated the density profiles as a function of the local distance to either the ice-liquid or liquid-vapor surfaces:

$$
z_{i, \alpha}^{*}=z_{i}-h_{\alpha}(x, y)
$$

where $\alpha=i w, w v$ stands here for either ice-water or water-vapor surfaces. Note that adopting a local definition of the distance to the interface, the slice over which the number of water molecules is counted is no longer planar, as in the usual calculation of the density profile. This is illustrated for positions measured relative to the ice-water surface in Figure 3.
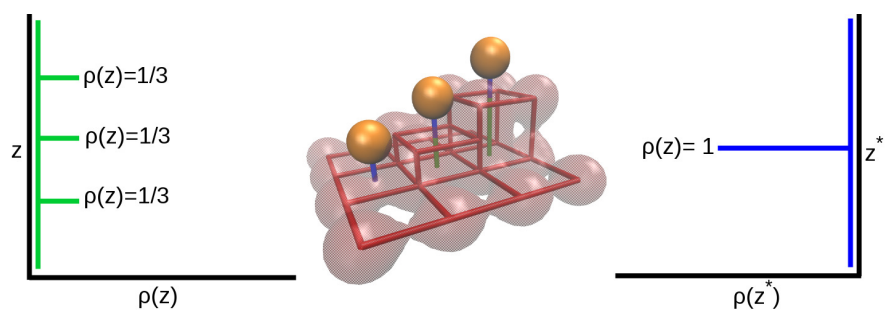

FIG. 3: Schematic illustration of the difference in the density profiles as a function of absolute $z$ coordinates (measured with respect to the simulation box reference frame) or as a function of the $z^{*}$ coordinate measured with respect to the local height of the ice-liquid interface. Pink faded spheres represent solid molecules, and orange spheres liquid molecules. The red grid shows the local ice-liquid interface. The three orange molecules are assigned to three different bins when measured in absolute values of $z$ (left scale), whereas to only one single bin when measured with respect to the local solid height (right scale).

\section{F. Estimation of water attachment rates}

Finally, we performed a last set of simulations aimed at measuring the attachment rate of water vapor to the ice surface. For this purpose, we performed the following experiment. A total of 400 water molecules were shot against 20 different thermal configurations of ice. Each water molecule shot was initially placed at a distance of $2 \mathrm{~nm}$ from the ice surface and assigned a velocity randomly chosen from the corresponding MaxwellBoltzmann distribution, with the initial $z$ component directed to the interface. The simulations were carried out in the microcanonical ensemble to avoid kinetic energy dissipation before and after the collision. We used a time step of $0.6 \mathrm{fs}$ in these simulations to ensure energy conservation. Using the initial velocity we estimate the time required for the water molecule to reach the surface in a ballistic trajectory, and allow the simulation to 
proceed for twice as much time. At the end of the simulation we perform a cluster analysis over all molecules and determine whether the colliding molecule is attached to the condensed phase or not. From the ensemble of trajectories, we can estimate the attachment rate $(\alpha)$, which is defined as the ratio between the water molecules that are incorporated into the ice surface after the impact $\left(N_{a d s}\right)$ and the total number of water molecules that are shot against the ice surface $\left(N_{\text {total }}\right): \alpha=N_{a d s} / N_{\text {total }}$. Notice that in this way we are measuring a direct attachment rate, not including the net rate due to evaporation events. These measurements were carried out at three different temperatures, $T=230,260$ and $270 \mathrm{~K}$ and at two nitrogen partial pressures, namely, zero and a 1 bar.

\section{RESULTS}

In this section we present results for the structure and attachment kinetics of ice in the presence of nitrogen. Our study covers a temperature range between $230 \mathrm{~K}$ to $270 \mathrm{~K}$, which spans most of the relevant temperature of ice crystal growth in the atmosphere as described in the Nakaya diagram. For the pressure, we have chosen conditions pertaining to the International Standard Atmosphere at those temperatures (c.f. I). This corresponds to pressures well above typical values in cirrus clouds, and therefore provide an upper limit for the influence of nitrogen on the ice surface. The detailed conditions studied in our simulations as well as the collected results may be found in Table III.

\section{A. Structure of the pristine ice/vapor interface}

A priori, the study of the ice/vapor interface is complicated by the presence of a thin layer of disordered ice, which grows as the temperature rises along the ice/vapor sublimation line.[60, 62] Depending on the community, this layer is known as a premelting film or quasi-liquid layer. Here we will stick to the standard terminology of wetting and adsorption physics, and denote the growing adsorbed layer as a premelting film.

Because of the complex structure of this interface, it is convenient to label surface molecules in terms of a suitable order parameter that can distinguish between water-like or disordered molecules and solid-like or ordered molecules.[79] This allows us to give a detailed account of the complex ice/vapor interface with reference to two simpler ice/water and water/vapor surfaces, and to plot separate solid-like and liquid-like density profiles of water molecules.[77, 78]

Figure 4 shows the density of solid-like and liquid-like molecules as a function of the perpendicular distance to the interface for both basal and $\mathrm{pI}$ facets in a temperature interval between 230 to $270 \mathrm{~K}$. At low temperature $(230 \mathrm{~K})$, the solid-like density profile (4(a) and 4(c)) reveals an ordered crystalline solid with high density peaks that correspond to complete bilayers within the bulk of the crystal (five for the basal facet and six for the pI facet 
in our simulation setup). Each such bilayer contains the molecules of full stacked hexagonal rings of ordered ice.[23-26, 62] As we move away from the bulk solid towards the vapor phase, we find a decay of the solid-like density profile. The interface consists of two partially formed bilayers of intermediate and small density. The outermost bilayer is indicated in the plot with a black vertical line.

As temperature increases towards the melting point, the height of the interfacial bilayers decreases smoothly up to $260 \mathrm{~K}$. However, in the range between $260 \mathrm{~K}$ and $270 \mathrm{~K}$, one full bulk like solid bilayer per interface melts into the premelting film. A similar bilayer melting has been observed previously for the TIP4P/ice model that is consistent with Sum Frequency Generation experiments, albeit at a somewhat lower temperature of about 250 K. $[62,85]$ The difference with previous simulations lies in the improved density representation, which reveals the bilayer melting process more clearly.

Interestingly, the structure of the solid like density profile at the interface remains qualitatively similar along the full temperature range studied, even after the first full bilayer melting (i.e., remains composed of two partial bilayers with intermediate and small densities). This process of melting can be observed also with the complementary density profiles of liquid-like molecules depicted in 4(b) and 4(d). In the range between $230 \mathrm{~K}$ and $260 \mathrm{~K}$ the profiles reveal an adsorbed film of water molecules which is made from three partial bilayers for the basal face and only two partial bilayers for the pI plane. As temperature rises in this range, the density of the profile increases slightly, but in the range between 260 to $270 \mathrm{~K}$, two of the partial bilayers attain bulk like densities, as found recently for the related TIP4P/2005 model.[77] The premelting film is never thick enough for the density of liquid molecules to attain a plateau value, but the maxima of the density peaks clearly attains order of magnitude values expected for bulk liquid water

The density profiles shown here describe the laterally averaged structure of the ice/vapor interface. A hint on the surface structure in the direction parallel to the interface is given by the partially filled density peaks found in the outermost layer. Their location remains congruent with the lattice spacing, but the height is significantly smaller than that observed in bulk. However, a difference is observed between basal and pI planes. In the former, the small peak is separated from a neighboring peak by a region of depleted density. In the latter, on the contrary, the peak rather appears as a shoulder and hardly exhibits a density minimum separating it from the next peak. This suggests that the outermost region of the ice surface, at least for the basal plane, exhibits interrupted regions of fully formed layers, leading to a stepped surface.[78] 
Basal

Prismatic I

\begin{tabular}{|c|c|c|c|c|c|c|c|c|c|c|}
\hline $\mathrm{T}$ & $\mathrm{p}$ & $\delta h$ & $\Gamma$ & $K$ & $\Delta \gamma$ & $\mathrm{p}$ & $\delta h$ & $\Gamma$ & $a$ & $\Delta \gamma$ \\
\hline K & $10^{-5} \mathrm{~Pa}$ & $\AA$ & $n m^{-2}$ & $10^{-11} \mathrm{~m} / \mathrm{J}$ & $m N / m$ & $10^{-5} \mathrm{~Pa}$ & $\AA$ & $n m^{-2}$ & ${ }^{11} \mathrm{~m} / \mathrm{J}$ & $m N / m$ \\
\hline 230 & 0.34 & 4.1 & 0.008 & 2.52 & -0.03 & 0.37 & 3.8 & 0.008 & 2.39 & -0.03 \\
\hline 230 & 0.67 & 4.4 & 0.016 & 2.52 & -0.05 & 0.72 & 3.8 & 0.017 & 2.39 & -0.06 \\
\hline 230 & 1.01 & 4.3 & 0.026 & 2.52 & -0.08 & 1.10 & 4.0 & 0.027 & 2.39 & -0.08 \\
\hline 240 & 0.44 & 4.8 & 0.010 & 2.18 & -0.03 & 0.49 & 4.1 & 0.010 & 2.00 & -0.03 \\
\hline 240 & 0.78 & 5.2 & 0.016 & 2.18 & -0.06 & 0.87 & 4.1 & 0.017 & 2.00 & -0.06 \\
\hline 240 & 0.94 & 5.1 & 0.019 & 2.18 & -0.07 & 1.05 & 4.1 & 0.021 & 2.00 & -0.07 \\
\hline 240 & 1.00 & 5.1 & 0.023 & 2.18 & -0.07 & 1.12 & 3.9 & 0.023 & 2.00 & -0.07 \\
\hline 250 & 0.54 & 5.6 & 0.011 & 1.92 & -0.04 & 0.56 & 4.9 & 0.012 & 1.89 & -0.04 \\
\hline 250 & 0.66 & 5.6 & 0.012 & 1.92 & -0.04 & 0.71 & 5.0 & 0.013 & 1.89 & -0.05 \\
\hline 250 & 1.03 & 5.6 & 0.018 & 1.92 & -0.07 & 1.05 & 5.0 & 0.020 & 1.89 & -0.07 \\
\hline 250 & 1.10 & 5.5 & 0.021 & 1.92 & -0.07 & 1.13 & 5.0 & 0.021 & 1.89 & -0.07 \\
\hline 250 & 1.16 & 5.5 & 0.024 & 1.92 & -0.08 & 1.17 & 5.0 & 0.022 & 1.89 & -0.08 \\
\hline 260 & 0.66 & 6.3 & 0.011 & 1.60 & -0.04 & 0.71 & 5.8 & 0.011 & 1.52 & -0.04 \\
\hline 260 & 0.82 & 6.1 & 0.015 & 1.60 & -0.05 & 0.88 & 5.6 & 0.013 & 1.52 & -0.05 \\
\hline 260 & 0.98 & 6.2 & 0.016 & 1.60 & -0.06 & 1.06 & 5.6 & 0.016 & 1.52 & -0.06 \\
\hline 260 & 1.05 & 6.2 & 0.017 & 1.60 & -0.06 & 1.13 & 5.6 & 0.017 & 1.52 & -0.06 \\
\hline 260 & 1.10 & 6.2 & 0.016 & 1.60 & -0.06 & 1.20 & 5.8 & 0.018 & 1.52 & -0.07 \\
\hline 270 & 0.76 & 8.9 & 0.012 & 1.44 & -0.04 & 0.81 & 8.3 & 0.012 & 1.45 & -0.04 \\
\hline 270 & 0.88 & 9.1 & 0.013 & 1.44 & -0.05 & 0.94 & 8.1 & 0.014 & 1.45 & -0.05 \\
\hline 270 & 1.00 & 9.3 & 0.015 & 1.44 & -0.05 & 1.07 & 8.4 & 0.015 & 1.45 & -0.06 \\
\hline 270 & 1.05 & 9.2 & 0.013 & 1.44 & -0.06 & 1.12 & 8.5 & 0.016 & 1.45 & -0.06 \\
\hline 270 & 1.10 & 9.1 & 0.015 & 1.44 & -0.06 & 1.17 & 8.2 & 0.018 & 1.45 & -0.06 \\
\hline
\end{tabular}

TABLE III: Summary of systems studied and collected results for premelting thickness, $\delta h$, nitrogen adsorption, $\Gamma$, Henry adsorption constant, $a$ and change in surface tension, $\Delta \gamma_{i v}$, as a function of temperature and pressure for basal (left) and primary prismatic (right) facets. The color code employed in the table labels defines the color code for the forthcoming figures. 


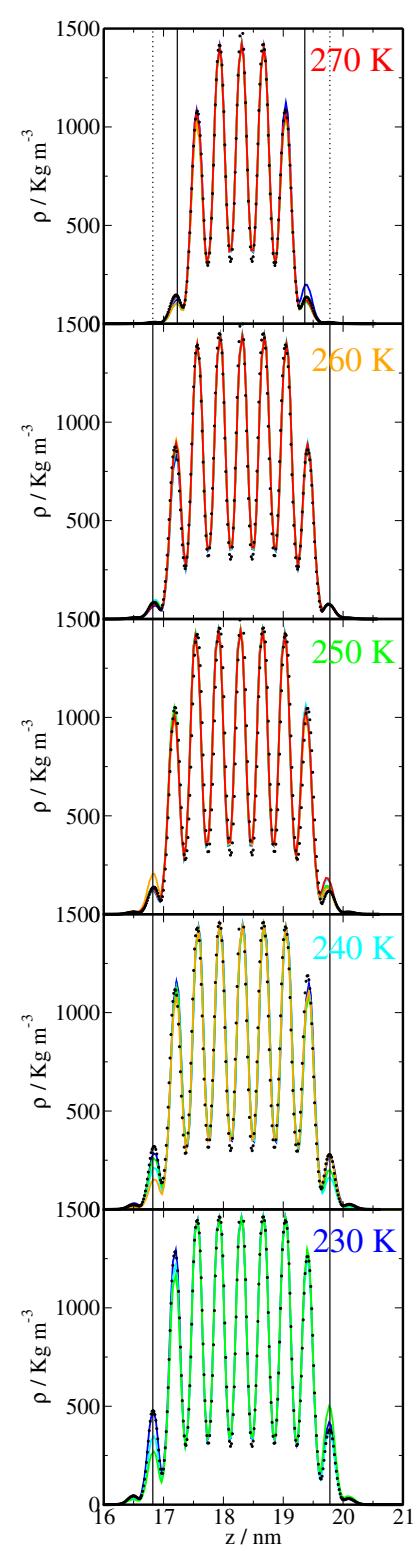

(a)basal

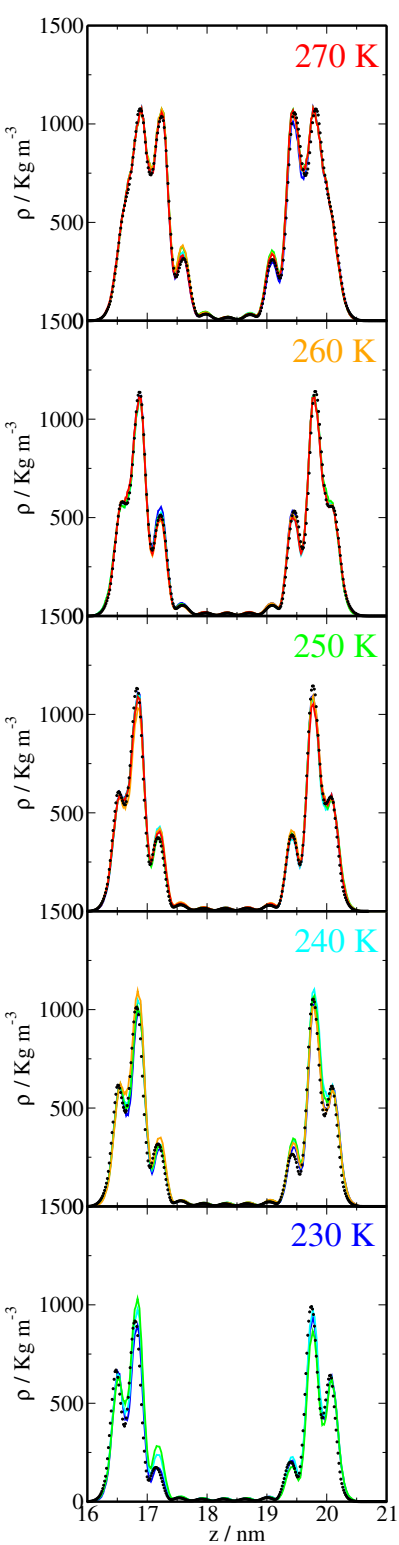

(b)basal

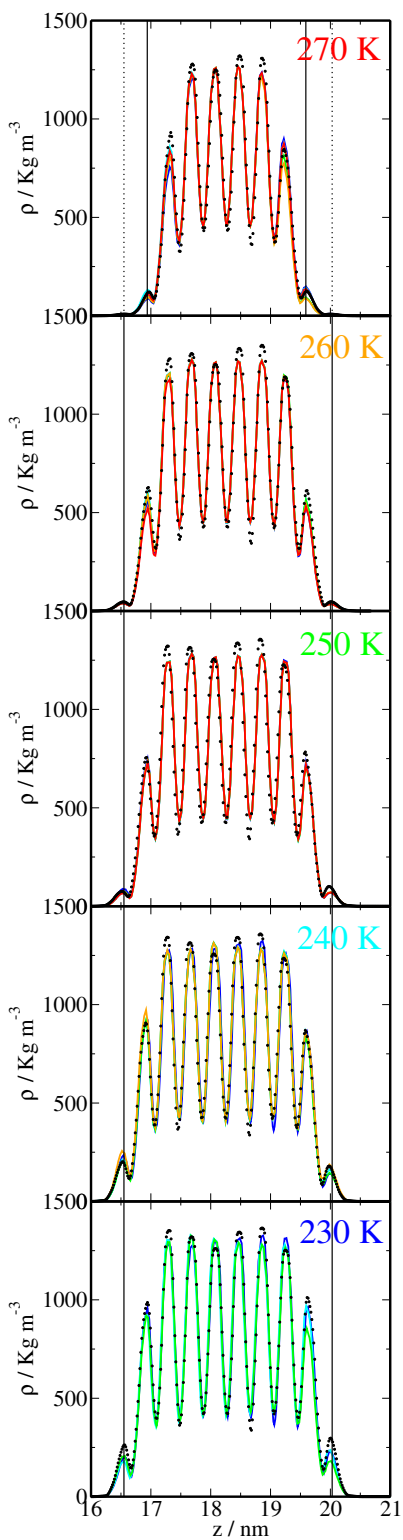

(c)pI

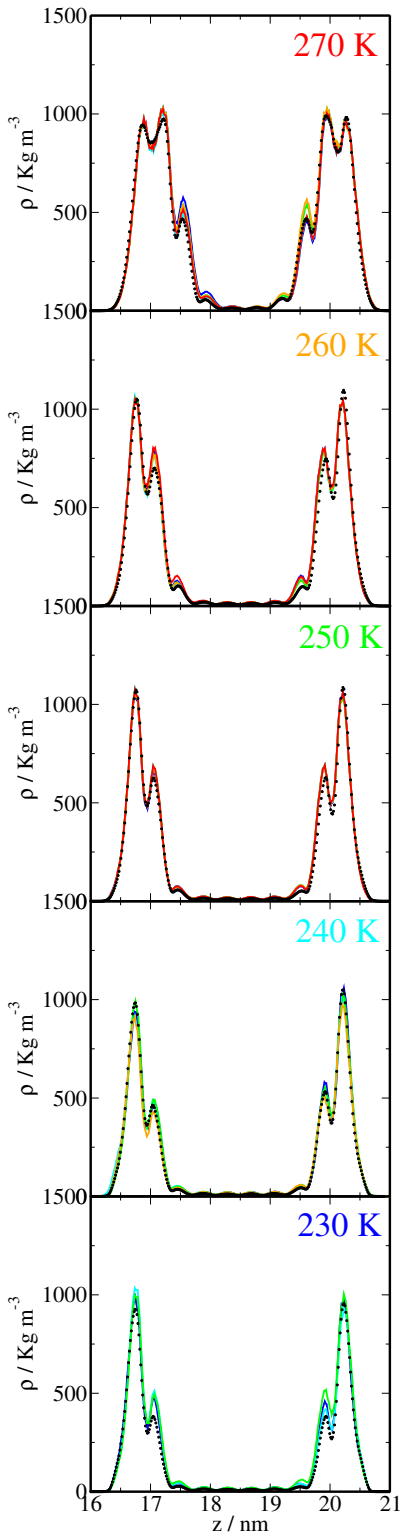

(d)pI

FIG. 4: Density profiles of water as a function of temperature and nitrogen pressure for basal (left) and primary prismatic (right) planes. Columns $(\mathrm{a}, \mathrm{c})$ correspond to density profiles of solid like molecules, while columns (b,d) correspond to density profiles of liquid like molecules. Black circles correspond to density profiles in pure water-vapor (zero nitrogen pressure), while colored lines stand for density profiles with cold to warm colors in order of increasing nitrogen pressure, with specific values of pressure as described in table III. For the density profile of solid like molecules, the full vertical line indicates the outermost interfacial bilayer. Notice how the location of this bilayer moves one lattice spacing towards the bulk phase at $\mathrm{T}=270 \mathrm{~K}$. 
In a forthcoming section, we study the attachment coefficient of water molecules on the ice surface. These are calculated by shooting water molecules against the ice substrate. Here, we exploit these data in order to obtain a scattering profile of the substrate. Merely by calculating the probability $P\left(z^{*}\right)$ to observe an impinging water molecule at a distance $z$ away from the average ice surface location, we find a revealing clue as to significant differences between basal and pI planes. Figure 5.a shows plots of $P\left(z^{*}\right)$ obtained from trajectories aimed at an ice substrate equilibrated under pure water vapor. The results show a region of slowly decaying probability at large $z$ that corresponds to close to ballistic trajectories of the water molecules, and then exhibits a high probability region corresponding to the sticking of the water molecules onto the ice substrate. Interestingly, for the basal face we find at the lowest temperature $\mathrm{T}=230 \mathrm{~K}$ a bimodal distribution, indicating that the water vapor molecules shot against the substrate have a choice of two preferred locations. The distance between the two maxima is about $0.25 \mathrm{~nm}$, which corresponds to the distance between adjacent planes in an ice bilayer (or alternatively, the perpendicular distance between the oxygen molecules of the stacked hexagonal rings). This bimodal structure smoothes at the temperature $\mathrm{T}=260 \mathrm{~K}$ and then eventually disappears at $\mathrm{T}=270 \mathrm{~K}$. For the pI plane, on the other hand, the distribution is unimodal for all temperatures studied, but clearly broadens as the temperature raises.

These results are clearly supportive of a stepped surface for the instantaneous configurations of basal facets, with stacks of half filled planes of half a bilayer and provide further evidence of the horizontally inhomogeneous distribution on the basal ice surface, as recently noted.[16, 18, 21] The structure of the pI facet on the contrary, does not appear to have this form of surface disorder, at least on the outermost liquid-vapor surface.
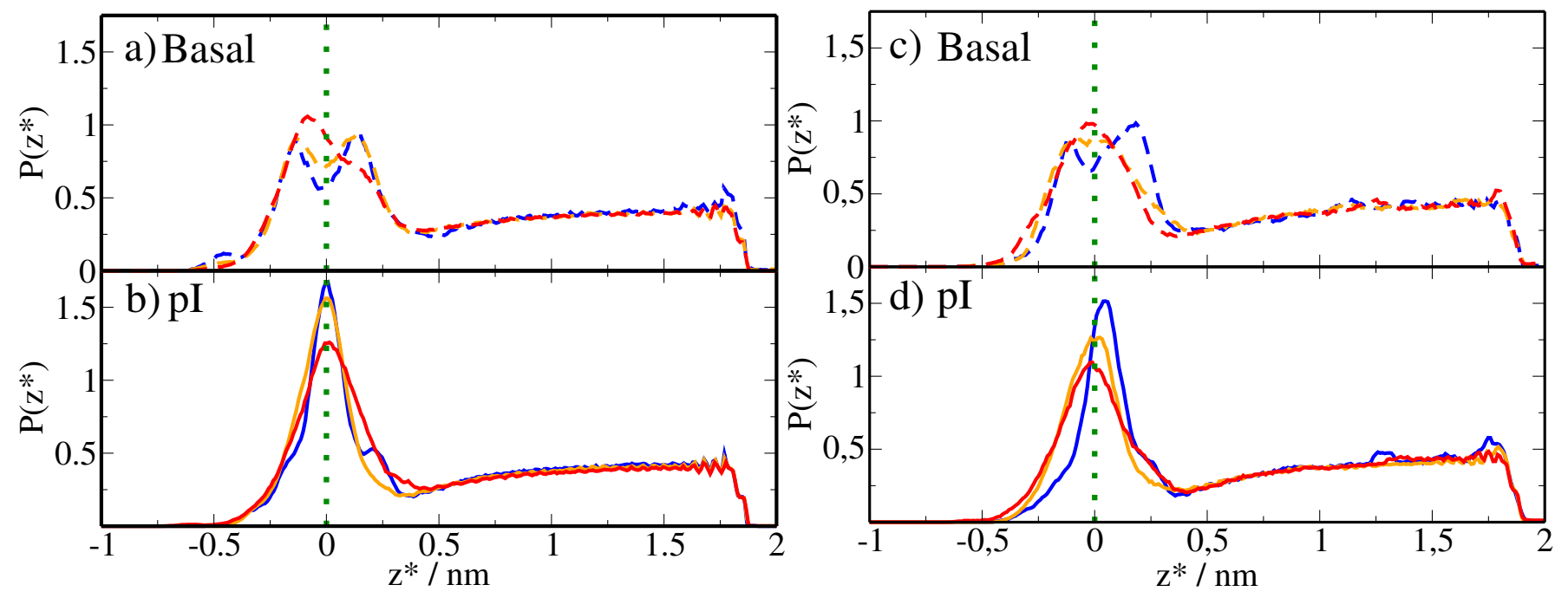

FIG. 5: Probability $P\left(z^{*}\right)$ of finding impinging water molecules at a distance $z$ for T=230 K (blue), 260 (orange) and 270 (red) at pressures of 0 bar $(a, b)$ and 1 bar $(\mathrm{c}, \mathrm{d})$. 


\section{B. Structure of the adsorbed nitrogen layer}

Having studied the structure of the pristine ice/vapor interface, we now consider the structure of the adsorbed nitrogen layer on the ice surface. Figure 6 shows the density profile of nitrogen molecules as a function of the perpendicular distance to the interface for both basal and pI planes. Results are given for temperature and nitrogen pressure made to match conditions of the International Standard Atmosphere for altitude in the range between 2500 and $9000 \mathrm{~m}$, which correspond to temperatures in the range 230 to $270 \mathrm{~K}$ studied above (c.f table I).

In all cases we find a plateau region of constant nitrogen density corresponding to the bulk gas phase, a small but significant adsorption layer at the interface, and a completely depleted central region corresponding to the highly nitrogen insoluble ice phase. Interestingly, the density profiles depicted here suggest a significant difference in the structure of adsorbed nitrogen at the basal and pI planes, with local densities that are roughly between twice (for the basal plane) and four times larger (for the pI plane) than the bulk vapor value. Also, the adsorption profile for the basal plane appears to be about twice as broad than the corresponding profile for the pI plane.

Unfortunately, these 'absolute' density profiles do not quite reveal the position of the adsorption peak relative to the complex ice/vapor interface. Particularly, it does not reveal whether the nitrogen is adsorbed on the premelting layer, or rather, is solubilized within. To study this issue further, we exploit the method of 'intrinsic' surfaces, which allows us to calculate local ice/film, $h_{i w}(\mathbf{x})$ and film/vapor, $h_{w v}(\mathbf{x})$ surfaces for instantaneous configurations during the simulation (c.f. section II D). From knowledge of the instantaneous local surfaces, we calculate so called 'intrinsic' density profiles, which are given as (c.f. 3):[86, 87]

$$
\rho_{\alpha}\left(z^{*}\right)=\left\langle\rho\left(z_{i}-h_{\alpha}(x, y)\right)\right\rangle
$$

where $\alpha$ is the surface label corresponding to either $w v$ or $i w$ surfaces.

Figure 7 shows the intrinsic density profile of nitrogen molecules as measured relative to the solid/liquid surface. In all cases, for both the basal and pI planes, the nitrogen adsorption peak is located at positive values of $z$, and reveals essentially zero nitrogen density within the bulk solid phase. Furthermore, the relative position of the adsorption peak moves away from zero as the temperature is raised. This is indicative of the growing premelting layer, which likely carries the adsorbed nitrogen away from the solid phase.

We can check this by plotting the intrinsic density profile of nitrogen molecules measured relative to the water/vapor surface, as shown in Figure 8(a). We find again that the adsorption peak lies always at positive $z$, outside the premelting layer. The difference is that now its location with respect to the film/vapor surface does not change with temperature. This confirms that the adsorbed nitrogen sticks on the film/vapor surface, and is carried away from the bulk solid as the premelting film grows. This behavior is similar to that observed for hydrocarbons and even far more soluble small organic molecules such as glyoxal, which have been observed to remains on the 
ice surface rather than sink into the premelting layer as ions do.[21, 22]

Also notice that only occasionally do we find finite nitrogen densities at negative $z$, whence, only a small fraction of all adsorbed nitrogen is dissolved into the premelting layer. A finite but very small amount of nitrogen may be seen to penetrate the premelting film for the pI plane at the two highest temperatures, but is negligible in most other cases.

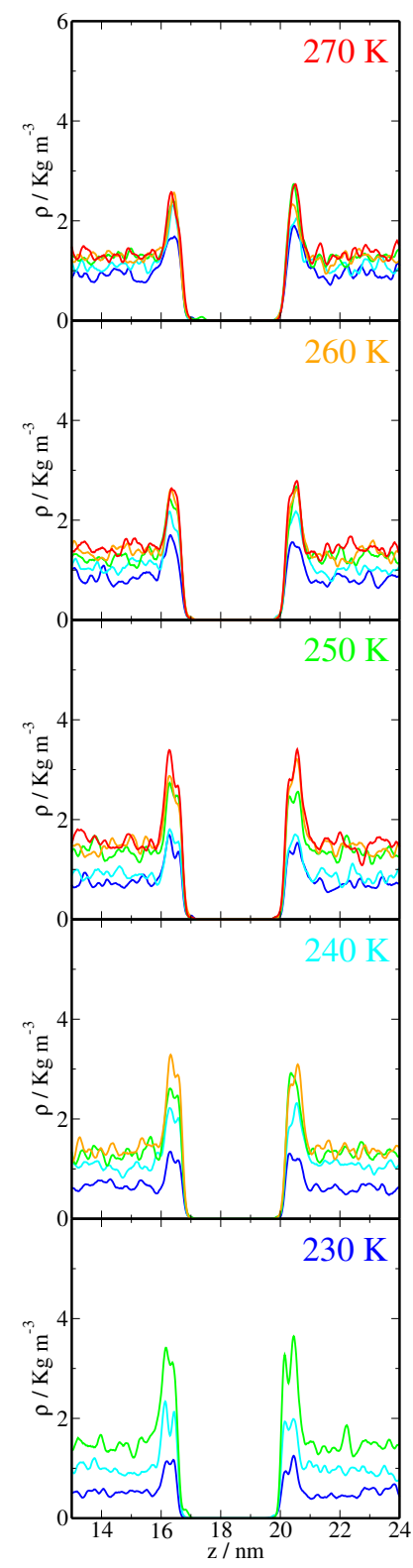

(a)basal

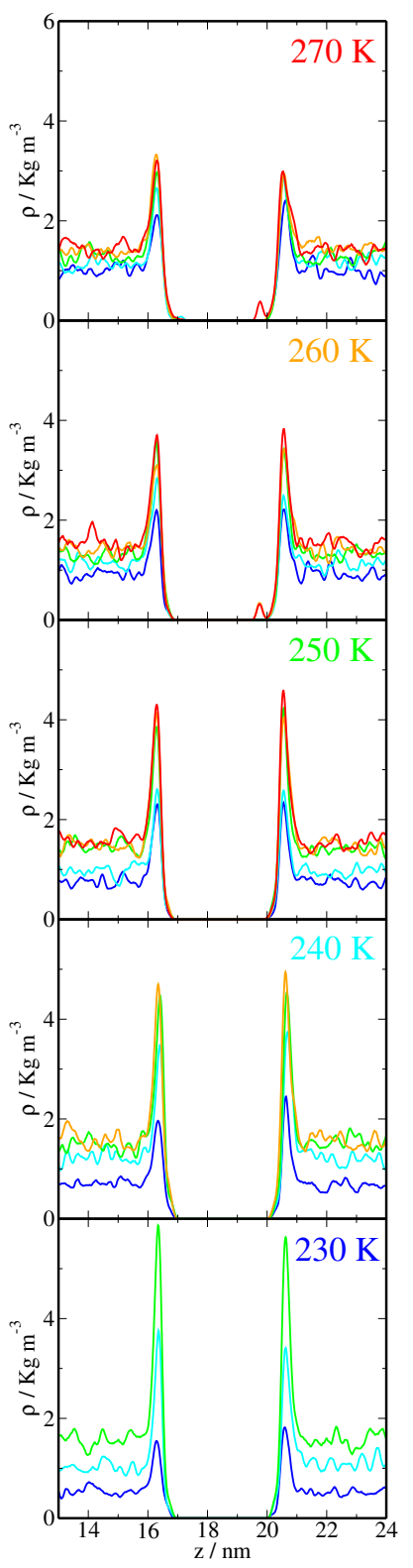

(b) $\mathrm{pI}$

FIG. 6: Density profiles of nitrogen as a function of perpendicular distance to the interface $z$. For each temperature, density profiles correspond to pressures as indicated in Table III, with pressure increasing in the range from ca. 0.3 to ca. 1 bar from cold to warm colors.

A striking feature of the intrinsic density profiles is that the adsorption peaks of both basal and pI planes are 
now very similar, exhibiting almost equal height and breadth. This means that at a given fixed point on either the water/vapor or ice/water surfaces, the nitrogen density profiles look just the same for both the basal and pI planes. The apparent differences in adsorption suggested by the absolute density profiles of Figure 6 is not really given by a distinct interaction of nitrogen with either plane, but rather, by a difference in the structure of the pristine crystal planes. The broader peak observed in the absolute density profiles for the basal plane is rather related to a surface structure with crystal steps and pockets, which can accommodate the nitrogen molecules at different absolute heights, as discussed previously. This reaffirms the observations of the inhomogeneity of the ice surface observed recently for the mW model.[16, 18, 21] 


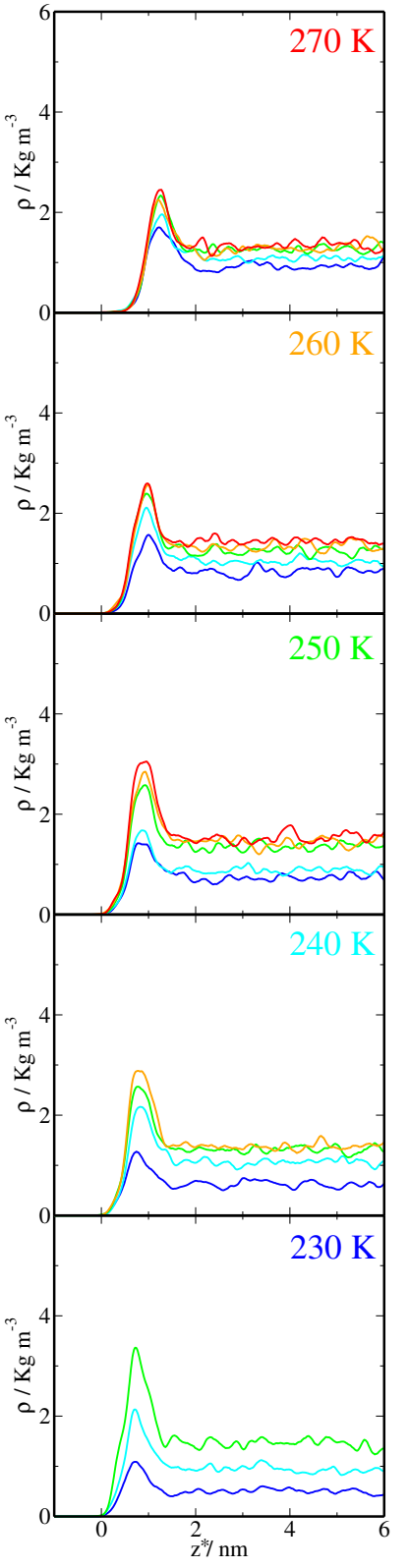

(a)basal

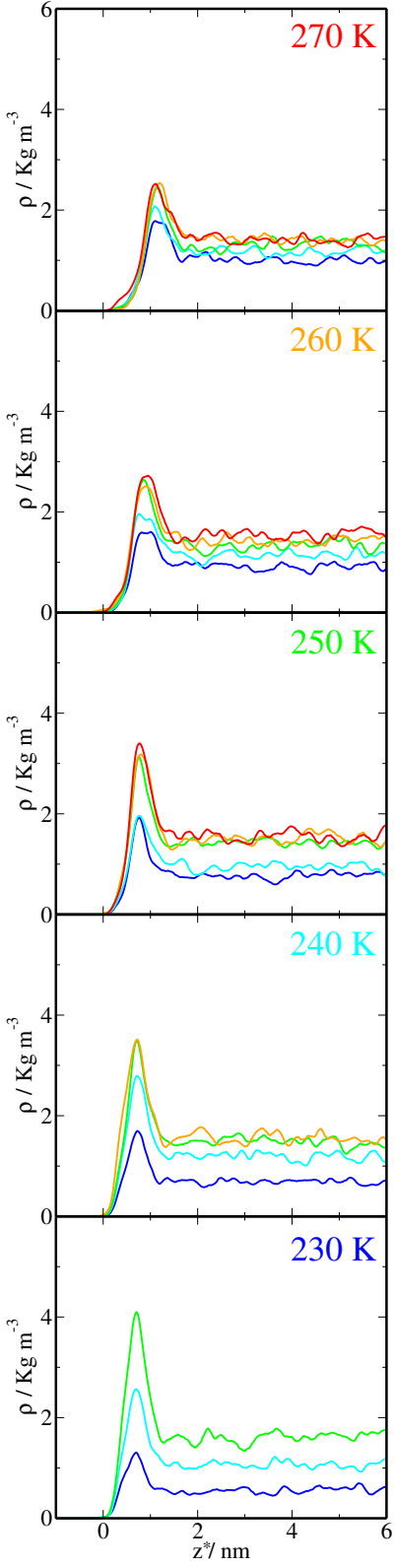

(b)pI

FIG. 7: Density profiles of nitrogen as a function of perpendicular distance to the ice-liquid surface $z-h_{i w}(x, y)$. For each temperature, density profiles correspond to pressures as indicated in Table III, with pressure increasing in the range from ca. 0.3 to ca. 1 bar from cold colors to warm colors. 


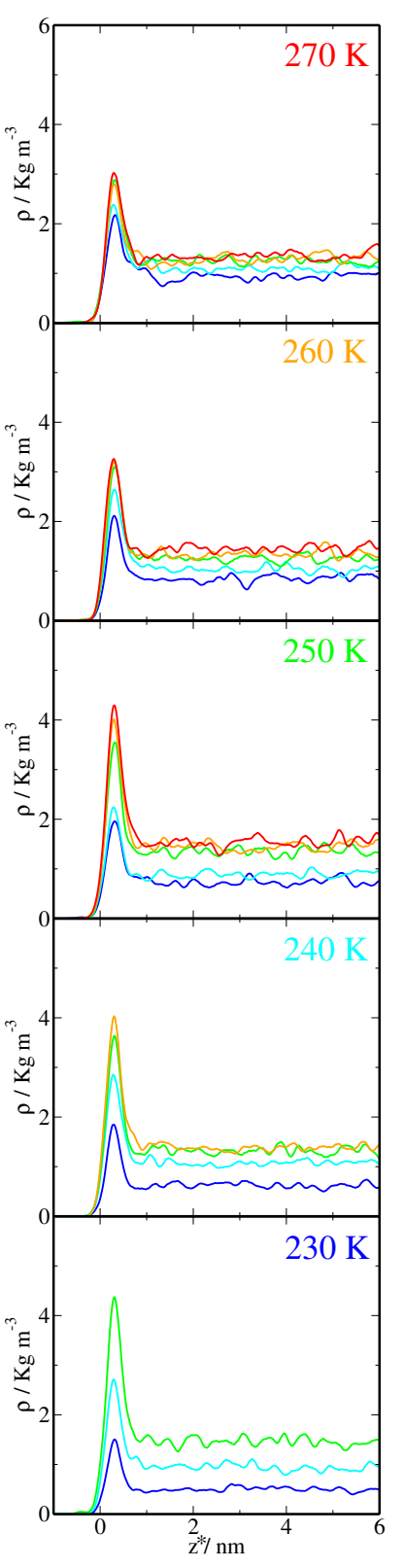

(a)basal

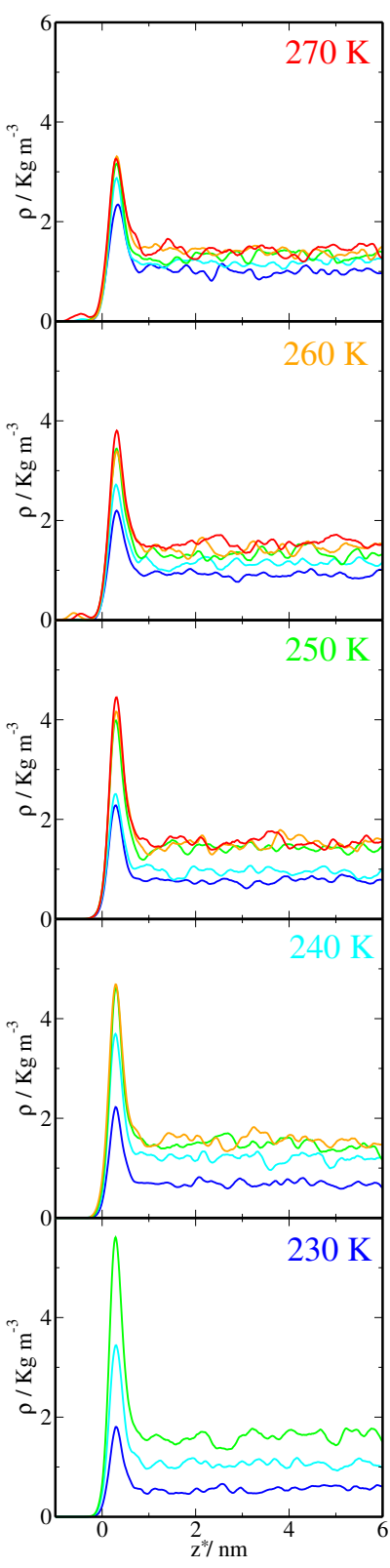

(b)pI

FIG. 8: Density profiles of nitrogen as a function of perpendicular distance to the liquid-vapor surface $z-h_{w v}(x, y)$. For each temperature, density profiles correspond to pressures as indicated in Table III, with pressure increasing in the range from ca. 0.3 to ca. 1 bar from cold colors to warm colors.

Interestingly, the picture that emerges for the ice surface in contact with low pressure nitrogen is similar to that observed for ice in contact with methane at 100 bar.[88] Despite this much higher pressure, the structure of the premelting layer remains unaffected by the influence of methane relative to the structure found in pure water vapor, provided results are compared for the same degree of under-cooling. In connection with the study of Shepherd et al., we expect that as the premelting layer becomes sufficiently thick, the solubility of nitrogen within the premelting film should become similar to that found in bulk water. However, here the partial pressures 
are very small, whence, the amount of adsorbed nitrogen can hardly be measured in our simulations.

\section{Structural and thermodynamic implications of nitrogen adsorption}

The study of nitrogen densities seems to indicate a rather passive role of the adsorbed nitrogen, which does hardly dissolve within the premelting film, but rather, remains adsorbed onto the disordered water layer. Furthermore, the surface enrichment is quite moderate, with a local density increase at the ice surface that is only a factor of two greater than the very small bulk gas density at atmospheric pressure.

To shed further light into this issue, we now look at the density of solid-like and liquid-like water molecules in the presence of nitrogen at different pressures (Figure 4). The results confirm that nitrogen gas does not result in any significant structural change of the ice/vapor interface. Indeed, neither the density of solid-like, nor the density of liquid-like water molecules seems to change with nitrogen pressure in any significant manner, for nitrogen pressures up to one bar. In fact, the density profiles corresponding to different nitrogen pressures can hardly be distinguished from the result of ice in pure water vapor.

Similarly, the plots of $P\left(z^{*}\right)$, obtained from the distribution of $\mathrm{H}_{2} \mathrm{O}(\mathrm{g})$ colliding against the substrate, remain also very similar to those observed in the absence of nitrogen, as shown in Figure 5.b. In this case, the distributions do not appear to be identical, but we attribute the small differences mainly to the small amount of equilibrated ice configurations employed in this study, which was just 20 , compared to the statistics gathered for the density profiles, which is taken over more than 400 configurations.

As a final illustration of the small role of nitrogen gas on the structure of the ice/vapor interface, we now concentrate on the influence of nitrogen gas on the thickness of the premelting layer, $\delta h$, calculated as described in section II D. Table III collects the results for all systems studied, while Figure 9 shows the premelting layer thickness as a function of nitrogen pressure for a set of temperatures in the range between $230 \mathrm{~K}$ and $270 \mathrm{~K}$. The results indicate that the premelting layer thickness does not show any significant change with nitrogen pressure, for pressures up to $1 \mathrm{bar}$, in all the temperature range studied, including the highest temperature of $270 \mathrm{~K}$. Notice that this corresponds to about $2 \mathrm{~K}$ less than the melting point of the TIP4P/ice model used here. The results are consistent with recent experiments, which indicate that the premelting film in presence of nitrogen remains in the subnanometer range up to $1 \mathrm{~K}$ below melting (similar to results found in pure water vapor, c.f.[41]), but increases very much in the scale of tenths of Kelvin away from the triple point.[27] This seems reasonable, since at such small distances away from the triple point the premelting film could thicken considerably and incorporate nitrogen and other impurities by dissolution. These small perturbations slightly change the intermolecular forces between the film and ice, but very close to the triple point such small changes in the intensity of the intermolecular forces can can significantly shift the equilibrium thickness.[63] 


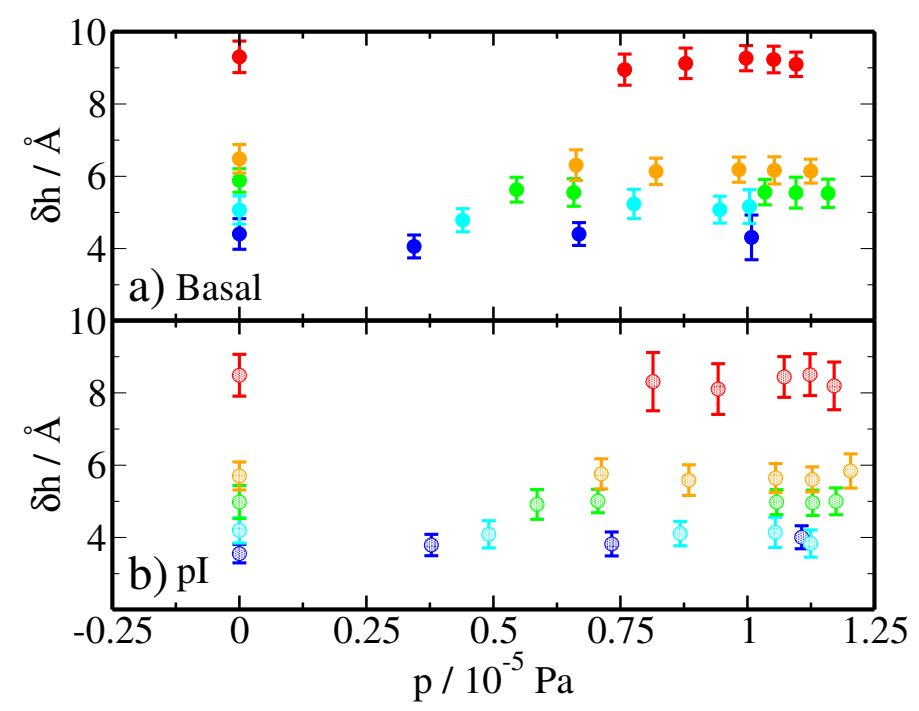

FIG. 9: Premelting layer thickness, $\delta h$, for basal and primary prismatic planes as a function of nitrogen pressure for $\mathrm{T}=230 \mathrm{~K}$ (blue), $240 \mathrm{~K}$ (cyan), $250 \mathrm{~K}$ (green), $260 \mathrm{~K}$ (orange) and $270 \mathrm{~K}$ (red).

As a summary, the structural analysis suggests that i) the ice/vapor surface essentially behaves as a high-energy inert substrate as regards nitrogen adsorption, with no significant disruption of the pristine interface and ii) there does not appear to be any apparent difference between the adsorption of nitrogen on basal or pI planes, other than that related with the differences between pristine interfaces under pure water vapor.

In order to quantify these qualitative statements, we now consider the thermodynamic implications of nitrogen adsorption on the ice/vapor interface. Firstly, we calculate the surface adsorption of nitrogen, $\Gamma_{\mathrm{N}_{2}}$, as given in terms of adsorbed molecules per unit surface (c.f.Eq. (2)).

Fig.10.a shows $\Gamma_{\mathrm{N}_{2}}$ as a function of nitrogen pressure for temperatures in the range 230 to $270 \mathrm{~K}$, for both basal and pI planes. Results obtained in a range of conditions relevant for most practical atmospheric conditions yield adsorptions that are essentially linear in the pressure, and correspond to a simple Henry adsorption law of the form $\Gamma_{\mathrm{N}_{2}}=a p$, with $a$, the Henry adsorption constant of nitrogen on ice. As regards the temperature dependence, the adsorption increases as temperature is decreased, as reflected by the slope of the adsorption isotherms. Overall, in all the regime of atmospheric conditions explored in this study, we find very small adsorption, and relatively small temperature and pressure dependences. Indeed, reported results for $\Gamma_{\mathrm{N}_{2}}$ hardly change by a factor of two from the highest to the lowest temperature, while the absolute values lie all in the order of $10^{-2}$ molecules per nanometer squared, whence, corresponding roughly to a surface concentration of one molecule per lateral area of $30 \times 30$ molecular diameters. This adsorption is however not small if we take into account the small solubility of nitrogen gas in water. Indeed, at the triple point the bulk solubility of nitrogen gas in water in terms of mole fractions is only $x_{\mathrm{N}_{2}} \approx 2 \cdot 10^{-5}$.[28, 89] This can be compared with an effective solubility of nitrogen on the premelting film, which we can estimate as $\chi_{\mathrm{N}_{2}}=\Gamma_{\mathrm{N}_{2}} A / N_{\mathrm{H}_{2} \mathrm{O}}$, and provides $\chi_{\mathrm{N}_{2}}=1.133 \cdot 10^{-4}$ and $1.125 \cdot 10^{-4}$ 
for basal and $\mathrm{pI}$ facets. Whence, the premelting film is enriched in nitrogen by an order of magnitude, although, as observed earlier, the nitrogen does not actually dissolve within, but rather, remains adsorbed atop the water/vapor surface. On the other hand, the maxima of the density profiles at the ice/vapor interface is between two and three times larger than the bulk gas density (c.f. Fig.6), but exhibits essentially no penetration into the bulk ice phase. Overall, the competition between partitioning and adsorption at the air/ice interphase is completely dominated by adsorption atop the premelting film.

In order to understand the thermodynamic significance of these results, we now use the adsorption constants to quantify the change in the ice/vapor surface tension resulting from the nitrogen adsorption at the interface. This can be estimated readily from the adsorption isotherms and the Gibbs adsorption equation (c.f. Eq. (3)-Eq. (4)). The results are shown in Figure 10.c, and clearly reveal an extremely small surface activity of nitrogen gas. The change in the ice/vapor surface tension resulting from the nitrogen adsorption is of order $-10^{-2} \mathrm{mN} / \mathrm{m}$, which is to be compared with the estimated ice/vapor surface tension, on the order of $100 \mathrm{mN} / \mathrm{m} .[90,91]$ Whence, nitrogen at conditions relevant to atmospheric sciences decreases the ice/vapor surface tension to a negligible extent. The results also reveal an extremely small surface anisotropy of the adsorption process, with only a slightly larger surface activity on the basal plane than on the pI plane.

To further characterize the energetics of nitrogen adsorption on the ice surface, we fit $\Gamma_{\mathrm{N}_{2}}$ to the linear law, $\Gamma_{\mathrm{N}_{2}}=a p$, and obtain adsorption constants from the the slope. These Henry's adsorption constants are expected to follow an Arrhenius behavior. This is tested and confirmed in Figure 10.c. From the slope of the Arrhenius plots, we find adsorption enthalpies of 7.4 and $6.5 \mathrm{~kJ} / \mathrm{mol}$ for the basal and pI surfaces, respectively (c.f. Table II). On the other hand, experimental measurements of nitrogen adsorbed on ice from BET isotherms yield an excess enthalpy of adsorption of about $2.5 \mathrm{~kJ} / \mathrm{mol}$ at $77 \mathrm{~K},[29-31]$ as measured relative to nitrogen enthalpy of condensation at $77 \mathrm{~K}$. Notice however that BET experiments report binding energies relative to the enthalpy of vaporization of nitrogen at $77 \mathrm{~K}$. From standard phase-change thermodynamic data available at the National Institute of Standards and Technology web page, we find an enthalpy of vaporization of $5.6 \mathrm{~kJ} / \mathrm{mol}$, which added to the $2.5 \mathrm{~kJ} / \mathrm{mol}$ excess enthalpy of adsorption yields $\Delta H_{a d s}=5.6+2.5=8.1 \mathrm{~kJ} / \mathrm{mol}$. Estimates obtained from the frequency shift of the $\mathrm{OH}$ dangling bond stretch upon adsorption at $96 \mathrm{~K}$, provide ca. $8.7 \mathrm{~kJ} / \mathrm{mol}$.[73], while TPD experiments provide ca. $9.6 \mathrm{~kJ} / \mathrm{mol}$.[34-36] However, as acknowledged, TPD probes only the high-value tail of the distribution of binding energies, so that the latter values are expected to be an upper bound for desorption energies at higher temperatures.[34-36] Since most of the conclusions of this work follow essentially from the low adsorption energies of nitrogen on ice, it follows that improvements on the force field employed will hardly change them in any significant way, since we provide adsorption energies that are barely $2.5 \mathrm{~kJ} / \mathrm{mol}$ below the upper conceivable bound of $9.6 \mathrm{~kJ} / \mathrm{mol}$.

Our estimate from the analysis of adsorption constants thus looks rather reasonable, and at any rate provides 
adsorption energies far smaller than the calculated binding energies of water ad-atoms on ice, which range from 30 to $70 \mathrm{~kJ} / \mathrm{mol}$.[92] 

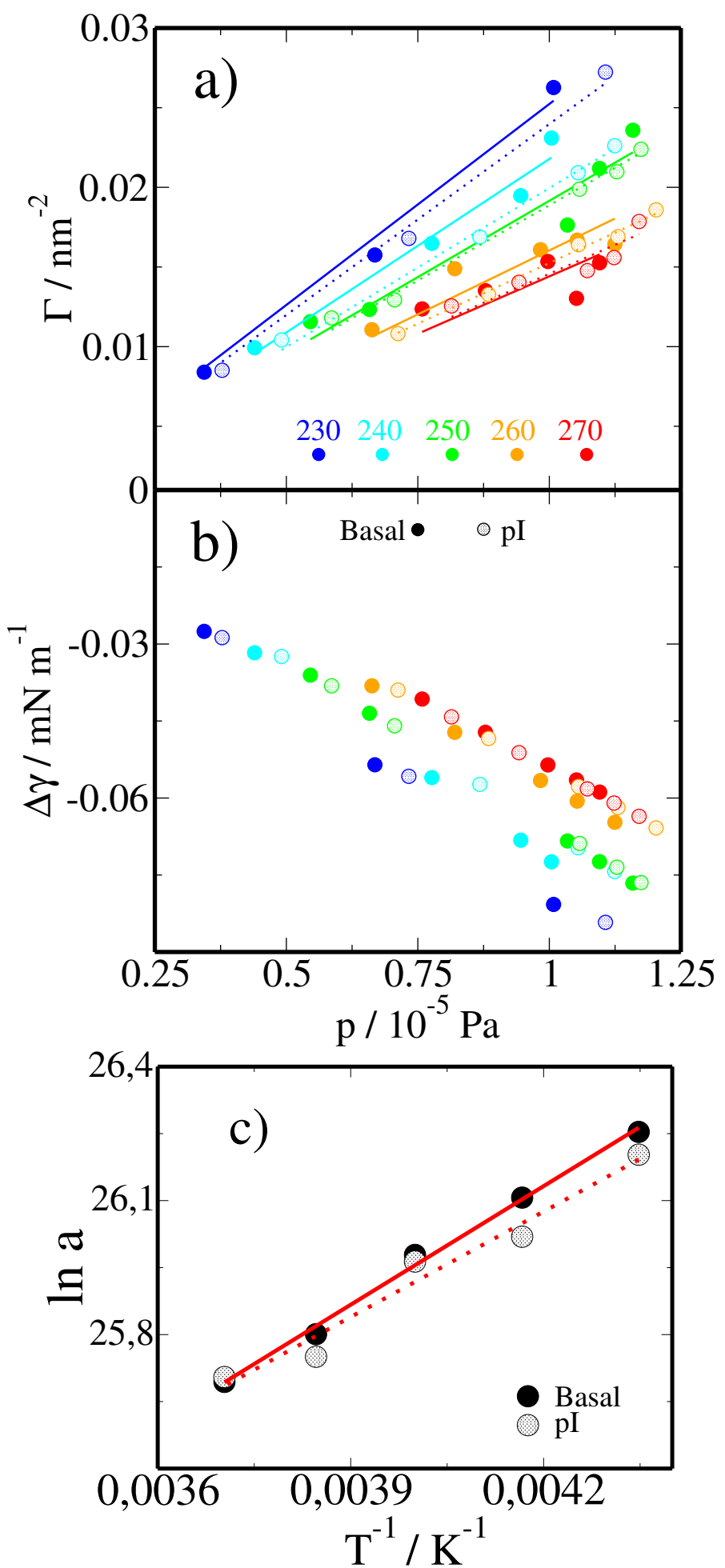

FIG. 10: Energetics of nitrogen adsorption. (a) Adsorption isotherms as a function of pressure, $\Gamma_{\mathrm{N}_{2}}$. (b) Change of the ice/vapor surface tension due to nitrogen adsorption, $\Delta \gamma$, as a function of pressure. (c) Arrhenius plots of Henry adsorption constants, $a$. Full symbols correspond to data for the basal plane, and empty symbols for the pI plane. Temperature color code as indicated in the legends on the plot. 


\section{SURFACE ATTACHMENT KINETICS}

In the previous sections, we have undertaken a study of the structure and thermodynamics of the ice surface in presence of nitrogen. The results reveal an almost negligible surface activity of nitrogen, and only a very small surface anisotropy as regards nitrogen adsorption. Accordingly, the reported influence of nitrogen gas on crystal growth rates must be related to kinetic effects.

Of course, significant amounts of crystal growth cannot be observed within the time scale of computer simulations. The success of the kinetic theory of gases and crystal growth theory is to write down the macroscopic net crystal growth rate in terms of microscopic properties that can be quantified without the need to monitor the process over macroscopic length and timescales.

In standard applications, the crystal growth rates are reported in terms of the maximum flux of water vapor molecules impinging onto the ice surface, which, from elementary kinetic theory is given as:

$$
J_{\max }=\frac{1}{4}\langle v\rangle \rho_{\mathrm{H}_{2} \mathrm{O}}
$$

where $\langle v\rangle$ is the thermal average speed of water molecules and $\rho_{\mathrm{H}_{2} \mathrm{O}}$ is the water vapor density in the neighborhood of the surface.

In practice, the crystal growth rate depends on the flux of vapor towards the surface, but also on the sublimation rate. Accordingly, the overall growth rate can be expressed as:

$$
R=\alpha J_{\max }-J_{e v}
$$

where $\alpha$ is the (microscopic) attachment coefficient, which dictates the probability that a waver molecule in a ballistic trajectory will stick to the ice surface, and $J_{e v}$ is a net flux of sublimation. In view of the difficulties to measure $\alpha$ and $J_{e v}$ separately, the crystal growth rate is usually given experimentally as:

$$
R=\alpha_{\text {eff }} J_{\max }
$$

where $\alpha_{\text {eff }}$ is now the (macroscopic) accommodation coefficient. This lumps into one single empirical parameter all the complicated surface kinetics, including the adsorption probability of impinging water molecules, the thermal equilibration and surface diffusion into the premelting film, the accommodation of a water-like molecule into the crystal lattice, and the decrease of the crystal growth rate due to sublimation events.[17, 93, 94]

A great number of experiments aimed at calculating $\alpha_{\mathrm{eff}}$ in atmospheric conditions have been performed, but unfortunately, results seem to depend considerably on experimental details and data analysis.[50, 93, 95-97] Most reported results vary between $\alpha_{\exp }=0.1$ to $\alpha_{\exp }=1$, but occasionally provide coefficients well smaller than 0.1 , leaving a great room for improvement.[98] 
One major source of discrepancy is related to the proper measurement of the net flux of impinging molecules. The reason is that this result is reliable only when the vapor density $\rho_{H_{2} O}$ is that of water vapor molecules at a distance from the surface in the order of the mean free path. Because most experiments are performed at ambient pressure, and necessitate an inert carrier gas to ensure thermal equilibrium, the macroscopic flow of water vapor is not related to ballistic trajectories implied in Eq. (9), but rather, is a slow random walk process. When the rate of attachment of water molecules into the surface is of the order of the rate of diffusion or larger, the density becomes considerably depleted at the neighborhood of the ice surface, and the calculation of $J_{\max }$ is then a complicated diffusion limited process. Disentangling this diffusion process from the attachment kinetics on the ice surface has revealed to be rather complicated and ambiguous.

An attractive way to disentangle the impingement rate from gas phase collision events is to carry out explicit computer simulations. In our study, we place water molecules with center of mass and angular velocities selected from a thermal Boltzmann distribution at a distance $2 \mathrm{~nm}$ away from an equilibrated ice surface, and shoot the water molecule against the surface, as explained in section IIF. Results for $\alpha$ collected over 400 trajectories and 20 different thermal ice configurations. are shown in Figure 11 at zero and one bar for three different temperatures. Detailed statistical information on the trajectories may be found in the supplementary material.

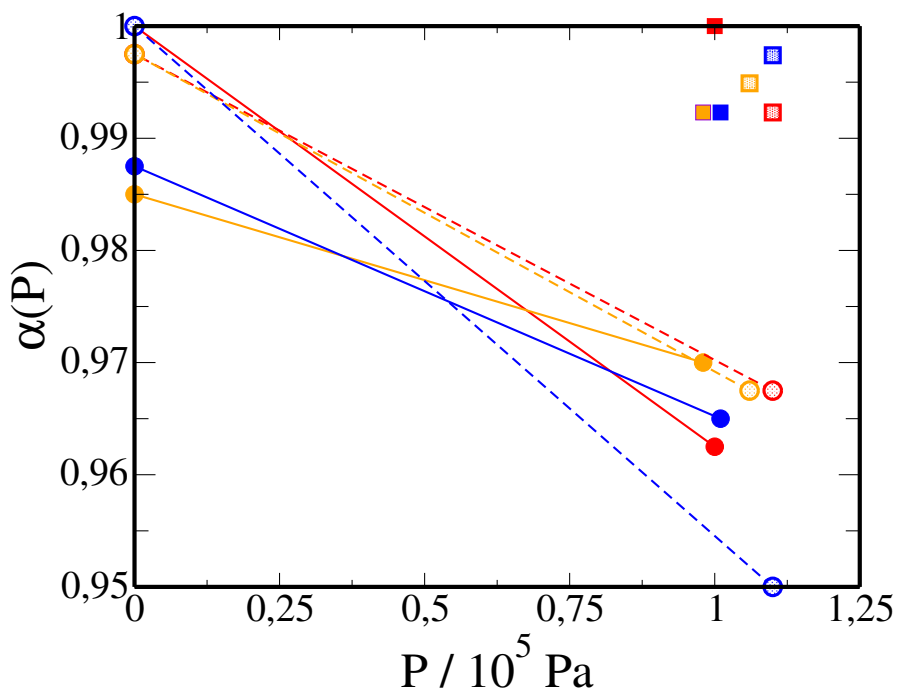

FIG. 11: Attachment coefficients at three different temperatures measured as a function of nitrogen partial pressure for $\mathrm{T}=230 \mathrm{~K}$ (blue), 260 (orange) and 270 (red). Filled circles correspond to data for the basal plane, and empty circles for the pI plane. Squares with similar color code show attachment coefficients calculated without counting trajectories that resulted in the back-scattering of water molecules with bulk nitrogen gas, as explained in the text.

At zero nitrogen pressure, the trajectories sampled are essentially ballistic, because the vapor pressure is extremely small for the TIP4P/ice model (this limitation is shared by most point charge models) and the probability of collisions with evaporated water vapor molecules is negligible for such short trajectories. The results of Fig- 
ure 11 show that this direct microscopic attachment coefficient is almost equal to unity for both the basal and pI surface planes. In fact, for the pI plane all impinging molecules were attached to the ice surface except one, at $\mathrm{T}=260 \mathrm{~K}$. For the basal plane $\alpha$ is also very close to unity, but five and six water molecules were reflected back at temperatures of $\mathrm{T}=230 \mathrm{~K}$ and $\mathrm{T}=260 \mathrm{~K}$, respectively. A look at the non-sticking trajectories shows that in these cases the reflection occurs only for gracing trajectories with a small z-component of the velocity. The molecule then flies at a small distance from the surface and is eventually reflected back by electrostatic repulsion at a distance (rather than in a hard-sphere like collision between molecules at close contact).

At a pressure of one bar, we find that $\alpha$ remains still very close to unity, but has decreased nevertheless significantly from the values at zero pressure. Out of 400 molecules shot against the ice/vapor interface, about 15 per batch were reflected and did not accommodate within the ice surface (see table IV of the supplementary material for detailed collision statistics). This remains a small number, but is about three times as large as in absence of nitrogen gas. In principle, the reason for the decrease in attachment rates could be 1) nitrogen disrupts the ice surface sufficiently that the attachment rates change 2) coverage of nitrogen on the ice surface is large, and water molecules are reflected by the adsorbed nitrogen 3) nitrogen gas bulk density is sufficient large that collisions in the bulk decrease the probability that water vapor molecules will reach the ice/vapor interface. Clearly, for the study of previous sections, the first two hypotheses seem unlikely: we have found that nitrogen barely changes the structure of the ice surface, and that the coverage of nitrogen corresponds to about one molecule every $100 \mathrm{~nm}^{2}$.

In order to shed further light into the problem, we reanalyzed the small set of trajectories which did not result in attachment of water and found that the overwhelming majority of trajectories resulted in reversal of the velocity component of the water molecules more than three molecular diameters away from the outer film/vapor surface (supplementary material, Table IV). Accordingly, it is concluded that the decrease of the attachment coefficients in presence of nitrogen is mainly due to collisions of water molecules with nitrogen gas from the bulk phase. To further illustrate the point, we calculate corrected attachment coefficients, using only those trajectories which do not involve scattering from gas phase nitrogen. The new results are shown in Fig.11 as squares, and are now fully consistent with the attachment coefficients found in absence of nitrogen. From this analysis we confirm that the active role of nitrogen gas is to reduce the rate at which water molecules can impinge on the ice surface, and is not related to a significant reduction of attachment probabilities of those water molecules that do collide with the surface.

The results for attachment coefficients at zero pressure are in full agreement with previous trajectory studies from molecular simulation, which have consistently observed that the fate of vapor water molecules directed towards the ice surface is to stick onto the ice surface,[94, 99, 100] irrespective of the rate of impingement.[13, 14] Our study of trajectories in the presence of nitrogen gas seem to indicate that a similar fate awaits water 
molecules unless an unfavorable collision with free nitrogen gas molecules occurs. Our results thus clearly favor (microscopic) attachment coefficients of unity, with little evidence of a significant temperature dependence. This is also consistent with some recent experiments for water vapor collision rates on ice.[96]

Why is there then so much scatter in the literature? Clearly, one reason is the difficulty to disentangle pure surface processes from gas phase diffusion processes. However, another large source of discrepancy likely lies in the definition of the accommodation coefficient itself. Here, we have tried to make clear the distinction between the surface attachment kinetics, which refers to the probability of a colliding molecule to stick onto the ice surface (which we term here the attachment coefficient, $\alpha$ ), from the effective accommodation coefficient, $\alpha_{\text {eff }}$ which accounts for the net balance between global condensation and evaporation kinetics as given in terms of the surface density. Further sources of confusion can arise if $\alpha_{\text {eff }}$ is measured in the diffusive regime with a flux $J_{\max }$ given in terms of the density at 'large' distances from the sample.[97] We believe the literature does not always clarify which is the actual coefficient that is measured, and this likely is one reason for the great scatter of experimental results.[98]

Additional support for a (microscopic) attachment coefficient of unity is given by the study of water gas molecules impinging onto super-cooled water. At least close to the triple point, where ice is expected to have a premelted liquid layer of nanometer size, $[10,77]$ the direct impingement of $\mathrm{H}_{2} \mathrm{O}(\mathrm{g})$ onto liquid water should not differ significantly, while precisely at the triple point, the effective coefficient should likewise be exactly the same, since then ice and water must have the same evaporation rates. The advantage of experiments performed in water is that the water/vapor interface here is much simpler in structure, so that one does not have to account for the complicated premelting mediated crystal growth mechanisms (c.f., $[13,93,101])$, and only the evaporation flux and diffusion processes needs to be considered. Despite of this somewhat simpler situation, attachment coefficients on liquid water are also difficult to measure and experiments exhibit a large scatter. However, by studying the uptake of a flux of deuterated water it is possible to disentangle the attachment rates from the evaporative flux, and the results obtained this way do seem to support a value of $\alpha=1,[102]$ in agreement with other recent experiments and computer simulations for attachment dynamics on liquid water.[103, 104]

\section{CONCLUSIONS}

In this paper we address the long standing controversy of nitrogen's role in determining ice crystal growth rates and premelting film thickness.[1, 27, 37-46, 49, 52, 54-59] Indeed, a large number of authors through the years have stressed the strong influence of either air or nitrogen in determining both the crystal growth rates, [37-39, 42] and the premelting layer thickness.[27, 40, 41, 58, 59] As far as crystal growth rates are concerned, two possible explanations have been put forward. 1) Either the nitrogen adsorption is strong enough to disrupt the ice surface 
and change the attachment kinetics, $[37-39,42]$ or 2) nitrogen plays a passive role merely by slowing down crystal growth rates due to a diffusion limited flow of water,[44-46, 49, 52, 54, 55] but unfortunately no consensus has been achieved from experiments.[37-39, 42, 44-46, 49, 52, 54, 55] As for the premelting layer thickness, impurities are suspected to influence the order of magnitude differences measured experimentally, but whether nitrogen is one such important factor remains unsolved.[27, 40, 41, 58, 59] Here we address these important controversies by performing computer simulation experiments with widely accepted point charge models of water and nitrogen. The direct measurement of nitrogen adsorption and attachment rates, as well as premelting layer thickness that can be performed by simulations give us the opportunity to unambiguously clarify these important problems of atmospheric science. To the best of our knowledge this is the first simulation work aimed at clarifying this long standing problem.

In our study we have carried out a detailed characterization of the ice surface in a controlled atmosphere of nitrogen gas. Results have been obtained at conditions found in the International Standard Atmosphere in a range of altitudes between 2750 to $9000 \mathrm{~m}$. This covers the relevant temperature range between 270 to $230 \mathrm{~K}$ expected for ice crystal growth in the atmosphere, and a pressure range between 0.5 and 1 bar well in the upper limit of relevant pressure for cirrus cloud formation.

From our study, we find i) the adsorbed amount of nitrogen gas on the ice surface is very small, corresponding to ca. one nitrogen molecule per $100 \mathrm{~nm}^{2}$. ii) nitrogen adsorbs essentially onto the premelting layer of ice and does hardly dissolve within. iii) The structure of the pristine ice/vapor surface is mediated by a thin liquid film, and does not change in any significant manner by the presence of small amounts of adsorbed nitrogen. iv) The thickness of the premelting layer in the temperature range studied varies between 0.4 to $1 \mathrm{~nm}$, and does not show any significant dependence on nitrogen pressure. v) The adsorption enthalpy of nitrogen on the ice/air interface is approximately $7 \mathrm{~kJ} / \mathrm{mol}$, much lower than is required for surface poisoning or step free energy changes to occur. vi) the variation of ice/air surface tension due to nitrogen is in the $10^{-2} \mathrm{mN} / \mathrm{m}$ range, and accounts for less than $0.05 \%$ of the full surface tension in pure water vapor. vii) Basal and pI surfaces of pristine ice exhibit significant differences. Particularly, the basal surface appears to show a disordered distribution of steps on the surface. However, nitrogen does not appear to shift this anisotropy in any significant manner. viii) Direct attachment rates of water molecules onto the ice surface are very close to unity, and do not change significantly by the presence of small amounts of adsorbed nitrogen. In essence, at temperatures relevant to ice formation and nitrogen pressures well in the upper limit of interest, the ice/vapor interface appears as a high-energy inert substrate for adsorbed nitrogen gas, which does not have any significant role on the structural properties of the ice/air interface, and the direct surface attachment kinetics. ix) However, our study shows conclusively that the nitrogen densities typical in the atmosphere are sufficiently large to deflect impinging water molecules directed to the ice surface from distances far smaller than the mean free path of water in pure vapor. This highlights the role of nitrogen gas in 
slowing down the flux of water vapor towards the crystal surface, and indicates that reported differences of crystal growth rate in a nitrogen atmosphere must be essentially related to diffusion limited processes.[37-39, 42] As a final comment, notice that the conclusions drawn here stem mainly from the low adsorption energies of nitrogen on ice. Since our own results provide an adsorption energy barely $2.5 \mathrm{~kJ} / \mathrm{mol}$ smaller than the upper bound of $9.6 \mathrm{~kJ} / \mathrm{mol}$ reported in Temperature Programmed Desorption experiments,[34-36] we expect that improvements on the force field employed here will not change our conclusions qualitatively.

\section{Acknowledgment}

We gratefully acknowledge funds from the Spanish Agencia Estatal de Investigación under Grant No. FIS2017-89361-C3-2-P.

[1] T. Bartels-Rausch, Nature, 2013, 494, 27-29.

[2] D. G. Loyola, S. Gimeno García, R. Lutz, A. Argyrouli, F. Romahn, R. J. D. Spurr, M. Pedergnana, A. Doicu, V. Molina García and O. Schüssler, Atmospheric Measurement Techniques, 2018, 11, 409-427.

[3] A. J. Baran, Atmospheric Research, 2012, 112, 45-69.

[4] S. G. Warren and R. E. Brandt, J. Geophys. Research, 2008, 113, D14220.

[5] E. Hesse, A. Macke, S. Havemann, A. Baran, Z. Ulanowski and P. Kaye, J. Quantitative Spectroscopy and Radiative Transfer, 2012, 113, $342-347$.

[6] S. Neshyba, B. Lowen, M. Benning, A. Lawson and P. M. Rowe, J. Geophys. Res.: Atmos., 2013, 118, 3309-3318.

[7] J. Voigtländer, C. Chou, H. Bieligk, T. Claus, S. Hartmann, P. Herenz, D. N. G. Ritter and F. S. Z. Ulanowski, Atmosph. Chem. Phys., 2018, 18, 13687-13702.

[8] E. Järvinen, O. Jourdan, D. Neubauer, B. Yao, C. Liu, M. O. Andreae, U. Lohmann, M. Wendisch, G. M. McFarquhar, T. Leisner and M. Schnaiter, Atmosph. Chem. Phys., 2018, 18, 15767-15781.

[9] J. P. D. Abbatt, Chemical Reviews, 2003, 103, 4783-4800.

[10] M. M. Conde, C. Vega and A. Patrykiejew, J. Chem. Phys., 2008, 129, 014702.

[11] A. J. Yang, M. Asta and B. B. Laird, Phys. Rev. Lett., 2013, 110, 096102.

[12] D. T. Limmer and D. Chandler, J. Chem. Phys., 2014, 141, 18 C505.

[13] S. Neshyba, J. Adams, K. Reed, P. M. Rowe and I. Gladich, J. Geophys. Res.: Atmos., 2016, 121, 14,035-14,055.

[14] A. Hudait and V. Molinero, J. Am. Chem. Soc., 2016, 138, 8958-8967.

[15] T. Kling, F. Kling and D. Donadio, The Journal of Physical Chemistry C, 2018, 122, 24780-24787. 
[16] I. Pickering, M. Paleico, Y. A. P. Sirkin, D. A. Scherlis and M. H. Factorovich, J. Phys. Chem. B, 2018, 122, 48804890.

[17] A. Mohandesi and P. G. Kusalik, J. Cryst. Growth, 2018, 483, 156 - 163.

[18] Y. Qiu and V. Molinero, J. Phys. Chem. Lett., 2018, 9, 5179-5182.

[19] P. Sandler, J. o. Jung, M. M. Szczesniak and V. Buch, J. Chem. Phys., 1994, 101, 1378-1391.

[20] C. Girardet and C. Toubin, Surf. Sci. Rep., 2001, 44, $159-238$.

[21] A. Hudait and M. T. A. V. Molinero, J. Am. Chem. Soc., 2017, 139, 10095-10103.

[22] A. Waldner, L. Artiglia, X. Kong, F. Orlando, T. Huthwelker, M. Ammann and T. Bartels-Rausch, Phys. Chem. Chem. Phys., 2018, 20, 24408-24417.

[23] G. Sazaki, S. Zepeda, S. Nakatsubo, M. Yokomine and Y. Furukawa, Proc. Nat. Acad. Sci., 2012, 109, 1052-1055.

[24] H. Asakawa, G. Sazaki, K. Nagashima, S. Nakatsubo and Y. Furukawa, Crystal Growth \& Design, 2015, 15, 33393344.

[25] H. Asakawa, G. Sazaki, K. Nagashima, S. Nakatsubo and Y. Furukawa, Proc. Nat. Acad. Sci., 2016, 113, $1749-1753$.

[26] K.-i. Murata, H. Asakawa, K. Nagashima, Y. Furukawa and G. Sazaki, Proc. Nat. Acad. Sci., 2016, 113, E6741E6748.

[27] T. Mitsui and K. Aoki, Phys. Rev. E, 2019, 99, 010801.

[28] R. Sander, Atmosph. Chem. Phys., 2015, 15, 4399-4981.

[29] A. W. Adams, L. M. Dormant and M. Orem, J. Colloid. Interface Sci., 1967, 25, 206-217.

[30] B. Schmitt, J. Ocampo and J. Klinger, J. Phys. Colloques, 1987, 48, C1-519-C1-525.

[31] J. T. Hoff, D. Gregor, D. Mackay, F. Wania and C. Q. Jia, Env. Sci. Tech., 1998, 32, 58-62.

[32] L. Hanot and F. Dominé, Env. Sci. Tech., 1999, 33, 4250-4255.

[33] L. Legagneux, A. Cabanes and F. Dominë, J. Geophys. Res.: Atmos., 2002, 107, ACH 5-1-ACH 5-15.

[34] E. C. Fayolle, J. Balfe, R. Loomis, J. Bergner, D. Graninger and M. R. K. I. Oberg, Astrophys J. Lett, 2015, 816, L28.

[35] M. Ninissale, E. Congiu and F. Dulieu, Astron. Astrophys., 2016, 585, A146.

[36] T. Nguyen, S. Baouche, E. Congiu, S. Diana, L. Pagani and F. Dulieu, Astron. Astrophys., 2018, 619, A111.

[37] W. Beckmann, J. Cryst. Growth, 1982, 58, 443 - 451.

[38] W. Beckmann, R. Lacmann and A. Bierfreund, J. Phys. Chem., 1983, 87, 4142-4146.

[39] T. Kuroda and T. Gonda, J. Met. Soc. Jap., 1984, 62, 563-572.

[40] M. Elbaum, S. G. Lipson and J. G. Dash, J. Cryst. Growth, 1993, 129, 491-505.

[41] H. Bluhm, D. F. Ogletree, C. S. Fadley, Z. Hussain and M. Salmeron, J. Phys.: Condens. Matter, 2002, 14, L227L233.

[42] K. G. Libbrecht, Annu.Rev.Mater.Res, 2017, 47, 271-295. 
[43] K. O. L. F. Jayaweera, J. Atmos. Sci., 1971, 28, 728-736.

[44] T. Kuroda and T. Gonda, J. Met. Soc. Jap., 1984, 62, 552-561.

[45] T. Gonda and M. Kombayashi, J. Met. Soc. Jap., 1970, 48, 440-450.

[46] D. Lamb and W. D.Scott, J. Cryst. Growth, 1972, 12, 21-31.

[47] W. Beckmann and R. Lacmann, J. Cryst. Growth, 1982, 58, 433-442.

[48] T. Gonda and T. Sei, J. Phys. Colloques, 1987, 48, C1-355-C1-359.

[49] T. Sei and T. Gonda, J. Cryst. Growth, 1989, 94, 697-707.

[50] K. G. Libbrecht and M. E. Rickerby, J. Cryst. Growth, 2013, 377, 1-8.

[51] N. Akutsu, Y. Akutsu and T. Yamamoto, Phys. Rev. B, 2001, 64, 085415.

[52] A. P. van den Heuvel and B. J. Mason, Nature, 1959, 184, 519-520.

[53] D. Lamb and P. V. Hobbs, J. Atmos. Sci., 1971, 28, 1506-1509.

[54] J. Hallett and B. J. Mason, Proc. R. Soc. Lond. A, 1958, 247, 440-453.

[55] T. Kobayashi, Physics of Snow and Ice: Proceedings, 1967.

[56] M. Elbaum, Phys. Rev. Lett., 1991, 67, 2982-2985.

[57] M. Elbaum and M. Schick, Phys. Rev. Lett., 1991, 66, 1713-1716.

[58] A. Lied, H. Dosch and J. H. Bilgram, Phys. Rev. Lett., 1994, 72, 3554-3557.

[59] H. Dosch, A. Lied and J. H. Bilgram, Surf. Sci., 1995, 327, 145-164.

[60] J. G. Dash, A. W. Rempel and J. S. Wettlaufer, Rev. Mod. Phys., 2006, 78, 695-741.

[61] Y. Li and G. A. Somorjai, J. Phys. Chem. C, 2007, 111, 9631-9637.

[62] A. Michaelides and B. Slater, Proc. Nat. Acad. Sci., 2017, 114, 195-197.

[63] J. Wettlaufer, Phys. Rev. Lett., 1999, 82, 2516-2519.

[64] H. Berendsen, D. van der Spoel and R. van Drunen, Comp. Phys. Comm., 1995, 91, 43 - 56.

[65] B. Hess, C. Kutzner, D. van der Spoel and E. Lindahl, J. Chem. Theo. Comp., 2008, 4, 435-447.

[66] G. Bussi, D. Donadio and M. Parrinello, J. Chem. Phys., 2007, 126, 014101.

[67] M. Allen and D. Tildesley, Computer Simulation of Liquids, Clarendon Press, Oxford, 2nd edn., 2017.

[68] D. Frenkel and B. Smit, Understanding Molecular Simulation, Academic Press, San Diego, 2nd edn., 2002.

[69] J. L. F. Abascal, E. Sanz, R. G. Fernandez and C. Vega, J. Chem. Phys., 2005, 122, 234511.

[70] J. J. Potoff and J. I. Siepmann, AIChE Journal, 2001, 47, 1676-1682.

[71] J. Sadlej, B. Rowland, J. P. Devlin and V. Buch, J. Chem. Phys., 1995, 102, 4804-4818.

[72] A. S. Tulegenov, R. J. Wheatley, M. P. Hodges and A. H. Harvey, J. Chem. Phys., 2007, 126, 094305.

[73] J. P. Devlin and V. Buch, J. Phys. Chem., 1995, 99, 16534-16548.

[74] C. Manca and A. Allouche, The Journal of Chemical Physics, 2001, 114, 4226-4234. 
[75] R. Estrada-Torres, G. A. Iglesias-Silva, M. Ramos-Estrada and K. R. Hall, Fluid Phase Equilibria, 2007, 258, 148 154.

[76] J. Rowlinson and B. Widom, Molecular Theory of Capillarity, Clarendon, Oxford, 1982.

[77] J. Benet, P. Llombart, E. Sanz and L. G. MacDowell, Phys. Rev. Lett., 2016, 117, 096101.

[78] J. Benet, P. Llombart, E. Sanz and L. G. MacDowell, Mol. Phys., 2019, 1-19.

[79] W. Lechner and C. Dellago, J. Chem. Phys., 2008, 129, 114707.

[80] P. J. Steinhardt, D. R. Nelson and M. Ronchetti, Phys. Rev. B, 1983, 28, 784-805.

[81] J. Benet, L. G. MacDowell and E. Sanz, J. Chem. Phys., 2014, 141, 034701.

[82] J. Benet, L. G. MacDowell and E. Sanz, Phys. Chem. Chem. Phys., 2014, 16, 22159-22166.

[83] J. R. Espinosa, C. Vega and E. Sanz, J. Phys. Chem. C, 2016, 120, 8068-8075.

[84] A. H. Nguyen and V. Molinero, J. Phys. Chem. B, 2014, 119, 9369 - 9376.

[85] M. A. Sánchez, T. Kling, T. Ishiyama, M.-J. van Zadel, P. J. Bisson, M. Mezger, M. N. Jochum, J. D. Cyran, W. J. Smit, H. J. Bakker, M. J. Shultz, A. Morita, D. Donadio, Y. Nagata, M. Bonn and E. H. G. Backus, Proc. Nat. Acad. Sci., 2017, 114, 227-232.

[86] M. Jorge, P. Jedlovszky and M. N. D. S. Cordeiro, J. Phys. Chem. C, 2010, 114, 11169-11179.

[87] M. Sega, B. Fabian and P. Jedlovszky, J. Chem. Phys., 2015, 143, 114709.

[88] T. D. Shepherd, M. A. Koc and V. Molinero, J. Phys. Chem. C, 2012, 116, 12172-12180.

[89] R. Battino, T. R. Rettich and T. Tominaga, J. Phys. Chem. Ref. Data, 1984, 13, 563-600.

[90] N. H. Fletcher, The Chemical Physics of Ice, Cambridge University Press, 1970.

[91] H. R. Pruppacher and J. D. Klett, Microphysics of Clouds and Precipitation, Springer, Heidelberg, 2010.

[92] M. Watkins, D. Pand, E. G. Wang, A. Michaelides, J. VandeVondele and B. Slater, Nature Materials, 2011, 10, 794-798.

[93] D. R. Haynes, N. J. Tro and S. M. George, J. Phys. Chem., 1992, 96, 8502-8509.

[94] S. Neshyba, E. Nugent, M. Roeselova and P. Jungwirth, J. Phys. Chem. C, 2009, 113, 4597-4604.

[95] C. Delval and M. J. Rossi, Phys. Chem. Chem. Phys, 2004, 6, 4665-4676.

[96] J. Skrotzki, P. Connolly, M. Schnaiter, H. Saathoff, O. Möhler, R. Wagner, M. Niemand, V. Ebert and T. Leisner, Atmosph. Chem. Phys., 2013, 13, 4451-4466.

[97] X. Kong, P. Papagiannakopoulos, E. S. Thomson, N. Markovië and J. B. C. Pettersson, J. Phys. Chem. A, 2014, 118, 3973-3979.

[98] IUPAC, Task Group on Atmospheric Chemical Kinetic DataEvaluation, http://iupac.pole-ether.fr, Iupac technical report, 2009.

[99] E. R. Batista, P. Ayotte, A. Bilić, B. D. Kay and H. Jónsson, Phys. Rev. Lett., 2005, 95, 223201. 
[100] W. Pfalzgraff, S. Neshyba and M. Roeselova, J. Phys. Chem. A, 2011, 115, 6184-6193.

[101] K.-i. Murata, K. Nagashima and G. Sazaki, Phys. Rev. Lett., 2019, 122, 026102.

[102] Y. Q. Li, P. Davidovits, C. E. Kolb and D. R. Worsnop, J. Phys. Chem. A, 2001, 105, 10627-10634.

[103] P. Davidovits, D. R. Worsnop, J. T. Jayne, C. E. Kolb, P. Winkler, A. Vrtala, P. E. Wagner, M. Kulmala, K. E. J. Lehtinen, T. Vesala and M. Mozurkewich, Geo. Phys. Res. Lett., 2004, 31,.

[104] A. Morita, M. Sugiyama, H. Kameda, S. Koda and D. R. Hanson, J. Phys. Chem. B, 2004, 108, 9111-9120. 
Supporting Information for

Structure and water attachment rates of ice in the atmosphere: role of nitrogen

by

\author{
P. Llombart, R. Bergua, E. G. Noya and L. G. MacDowell \\ Instituto de Química Física Rocasolano, CSIC, Calle Serrano 119, 28006 Madrid, Spain \\ and
}

Departamento de Química Física, Facultad de Ciencias Químicas, Universidad Complutense, Madrid, 28040, Spain.

\begin{tabular}{|c|c|c|c|c|c|c|c|c|c|c|c|c|c|c|c|c|c|c|c|c|}
\hline$\rho$ & $\mathrm{T}$ & $\mathrm{P}$ & $\rho$ & $\mathrm{T}$ & $\mathrm{P}$ & $\rho$ & $\mathrm{T}$ & $\mathrm{P}$ & $\rho$ & $\mathrm{T}$ & $\mathrm{P}$ & $\rho$ & $\mathrm{T}$ & $\mathrm{P}$ & $\rho$ & $\mathrm{T}$ & $\mathrm{P}$ & $\rho$ & $\mathrm{T}$ & $\mathrm{P}$ \\
\hline \multirow{9}{*}{0.56} & 230 & 0.39 & \multirow{9}{*}{0.79} & 230 & 0.55 & \multirow{9}{*}{1.03} & 230 & 0.71 & \multirow{9}{*}{1.26} & 230 & 0.87 & \multirow{9}{*}{1.50} & 230 & 1.01 & \multirow{9}{*}{1.73} & 230 & 1.14 & \multirow{9}{*}{1.96} & 230 & 1.25 \\
\hline & 235 & 0.40 & & 235 & 0.55 & & 235 & 0.70 & & 235 & 0.88 & & 235 & 0.99 & & 235 & 1.17 & & 235 & 1.35 \\
\hline & 240 & 0.39 & & 240 & 0.57 & & 240 & 0.72 & & 240 & 0.88 & & 240 & 1.08 & & 240 & 1.24 & & 240 & 1.37 \\
\hline & 245 & 0.42 & & 245 & 0.59 & & 245 & 0.74 & & 245 & 0.91 & & 245 & 1.08 & & 245 & 1.25 & & 245 & 1.42 \\
\hline & 250 & 0.42 & & 250 & 0.58 & & 250 & 0.75 & & 250 & 0.94 & & 250 & 1.11 & & 250 & 1.29 & & 250 & 1.42 \\
\hline & 255 & 0.42 & & 255 & 0.59 & & 255 & 0.78 & & 255 & 0.94 & & 255 & 1.09 & & 255 & 1.29 & & 255 & 1.45 \\
\hline & 260 & 0.43 & & 260 & 0.60 & & 260 & 0.80 & & 260 & 0.95 & & 260 & 1.16 & & 260 & 1.34 & & 260 & 1.48 \\
\hline & 265 & 0.44 & & 265 & 0.62 & & 265 & 0.80 & & 265 & 1.00 & & 265 & 1.11 & & 265 & 1.33 & & 265 & 1.52 \\
\hline & 270 & 0.46 & & 270 & 0.64 & & 270 & 0.82 & & 270 & 1.02 & & 270 & 1.19 & & 270 & 1.39 & & 270 & 1.55 \\
\hline
\end{tabular}

TABLE I: Equation of State for nitrogen. Pressure (P) is expressed in units of $10^{5} \mathrm{~Pa}$, temperature (T) in K and density $(\rho)$ in $\mathrm{kg} \mathrm{m}^{-3}$. 


\begin{tabular}{cc}
\hline \hline $\mathrm{T}$ & $B_{2}(T)$ \\
$K$ & $10^{3} \mathrm{~kg}^{-1} \mathrm{~m}^{3}$ \\
\hline 270 & -3.2 \\
265 & -11.9 \\
260 & -5.9 \\
255 & -13.8 \\
250 & $-4.5^{*}$ \\
245 & $-3.4^{*}$ \\
240 & $-4.7^{*}$ \\
235 & -13.0 \\
230 & -20.9 \\
\hline
\end{tabular}

TABLE II: Second virial coefficient $B_{2}$ as a function of temperature. ${ }^{*}$ Three out layers were not included in the fit for $B_{2}(T)$. 


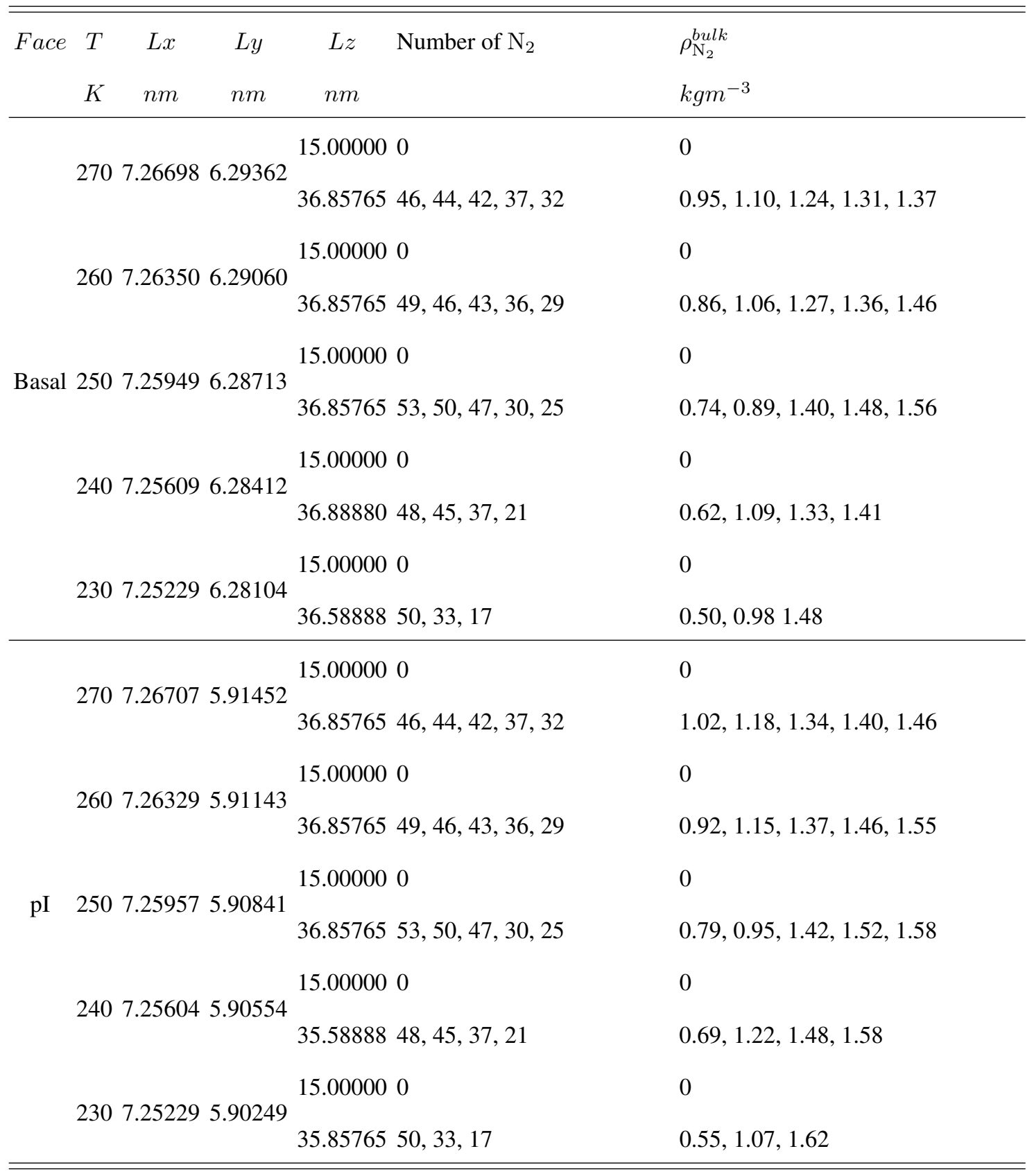

TABLE III: Summary of thermodynamic conditions and system sizes for the simulations of the ice interface in presence of nitrogen. 


\begin{tabular}{cccccc}
\hline \hline Facet $T / \mathrm{K} \mathrm{N}_{N_{2}}$ & $\mathrm{~N}_{\mathrm{ns}}$ & $\mathrm{N}_{e v}$ & $\alpha$ \\
\hline Basal & 270 & 42 & $14(14)$ & 2 & 0.9650 \\
Basal & 270 & 0 & 0 & 0 & 1.0000 \\
Basal & 260 & 43 & $12(9)$ & 2 & 0.9700 \\
Basal & 260 & 0 & 6 & 1 & 0.9850 \\
Basal & 230 & 50 & $14(11)$ & 0 & 0.9650 \\
Basal & 230 & 0 & 5 & 0 & 0.9875 \\
pI & 270 & 42 & $13(10)$ & 2 & 0.9675 \\
pI & 270 & 0 & 1 & 1 & 0.9975 \\
pI & 260 & 43 & $13(11)$ & 2 & 0.9675 \\
pI & 260 & 0 & 1 & 0 & 0.9975 \\
pI & 230 & 50 & $20(19)$ & 0 & 0.9500 \\
pI & 230 & 0 & 0 & 0 & 1.0000 \\
\hline \hline
\end{tabular}

TABLE IV: Table with detailed information of the collision statistics. $N_{N_{2}}$ is the number of nitrogen molecules present in the simulation box. $\mathrm{N}_{n s}$ is the number of water molecules shot a distance of $2 \mathrm{~nm}$ away from the surface and not sticking into the surface. Shown in parenthesis is the number of molecules which were reflected back to the gas phase by collisions with nitrogen gas molecules. $\mathrm{N}_{e v}$ provides the number of evaporation events observed during the simulations. 

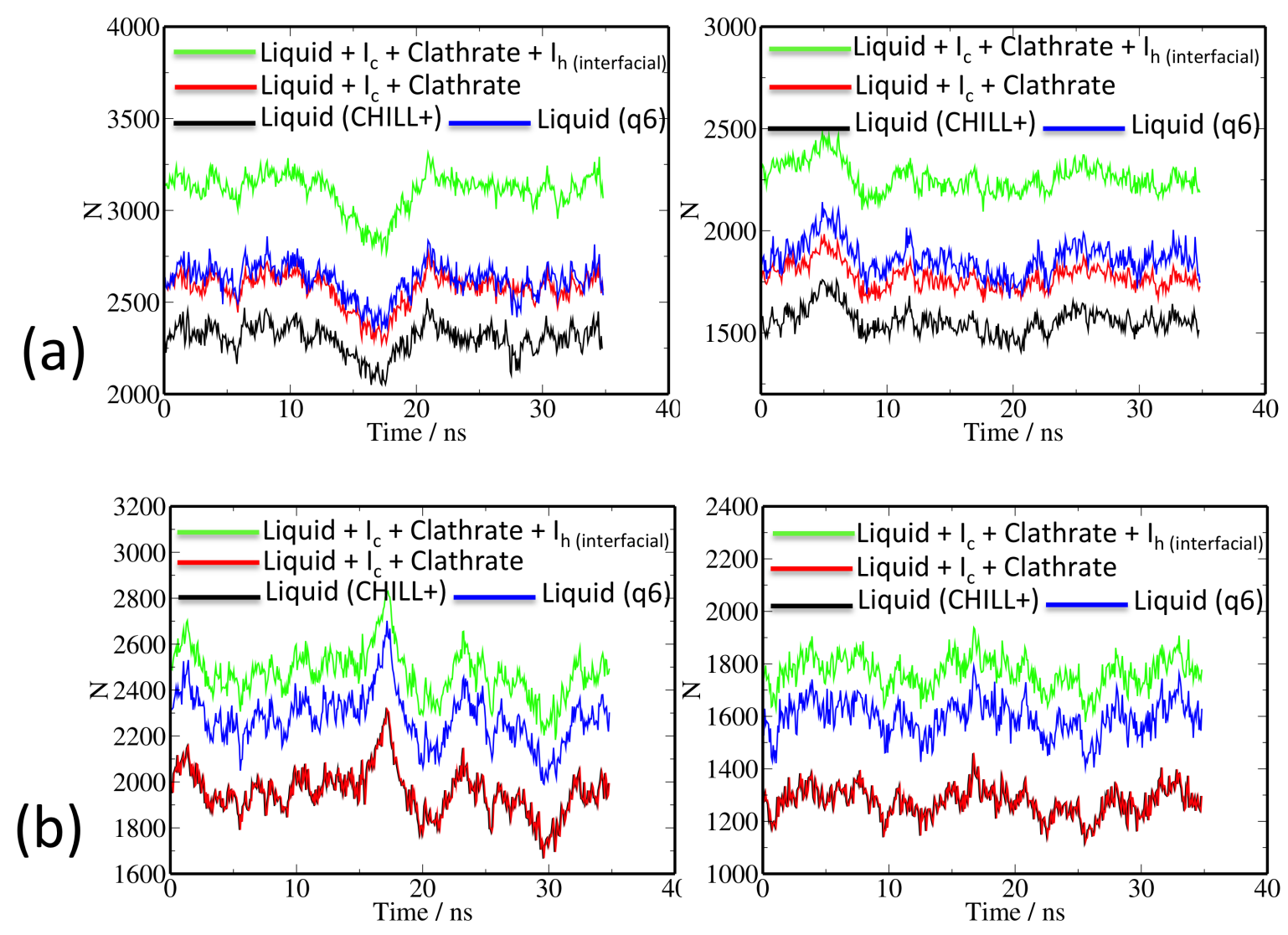

FIG. 1: Comparison of the number of liquid-like molecules as determined from the $\bar{q}_{6}$ parameter and the CHILL+ algorithm. ${ }^{84}$. a) Results for the Basal plane. b) Results for the prismatic plane. Plots are shown from left to right at 270, 260, 250, 240 and $230 \mathrm{~K}$, respectively. Blue: Runing number of liquid molecules during a simulation as obtained from the $\bar{q}_{6}$ parameter used in this work. Black: Runing number of liquid molecules as a function of time as extracted with the CHILL+ algorithm. Notice that the blue and black lines run almost parallel to each other, with a constant offset of about $12 \%$. Other possible choices to determine the thickness of the premelting layer remain also largely correlated. Red: Runing number of molecules in liquid, cubic and clathrate like environments as obtained from the CHILL+ algorithm. Green: Runing number of molecules in liquid, cubic, clathrate, and interfacial hexagonal environments as obtained from the CHILL+ algorithm. 

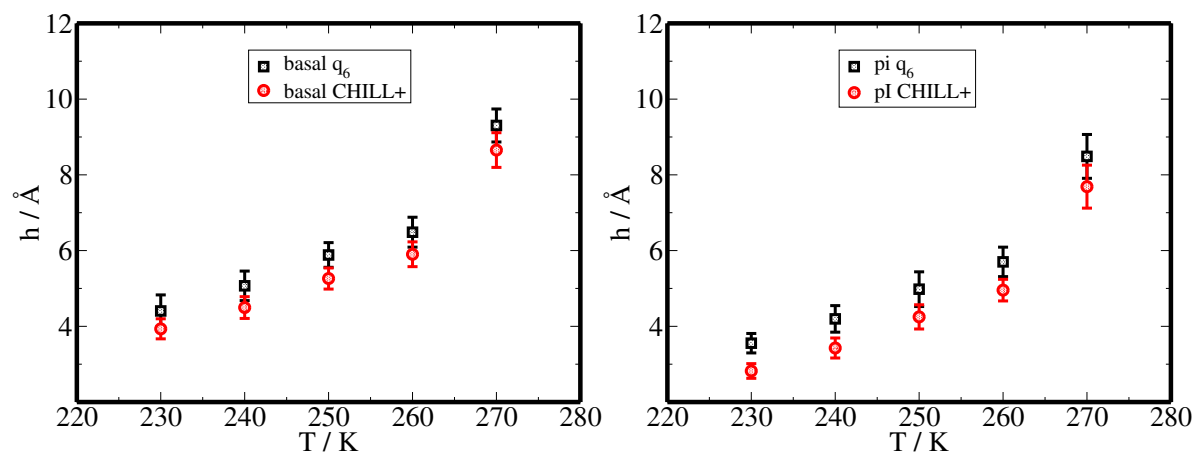

FIG. 2: Left: Basal. Right: pI. Premelting thickness at zero nitrogen pressure as calculated using the number of liquid like molecules from $\bar{q}_{6}$ (squares) used in this work and the CHILL+ algorithm (circles). Notice that the calculation of film heights differs by an almost constant offset. 